\title{
Title: Antidepressant treatment modulates the gut microbiome and metabolome during pregnancy and lactation in rats with a depressive-like phenotype
}

\section{Affiliations:}

$6 \quad{ }^{1}$ Department of Neurobiology, Groningen Institute for Evolutionary Life Sciences, University of

7 Groningen, Groningen, The Netherlands

$8 \quad{ }^{2}$ Center for Host-Microbial Interactions, ${ }^{3}$ Department of Biomedical Sciences, School of Veterinary

9 Medicine and Perelman School of Medicine, University of Pennsylvania, Philadelphia, PA 19104, USA

$10{ }^{4}$ Department of Pharmacology, Center for Epigenetic Research in Child Health and Brain Development,

11 University of Maryland School of Medicine, Baltimore MD 21201, USA

16 Correspondence to:

17 Jocelien DA Olivier, PhD

18 Groningen Institute for Evolutionary Life Sciences

19 University of Groningen

$20 \quad$ Nijenborgh 7

219747 AG Groningen

22 The Netherlands

23 Tel: +31503637221

24 Email: j.d.a.olivier@rug.nl 


\section{Abstract}

27 Background: Up to $10 \%$ of women use selective serotonin reuptake inhibitor (SSRI) antidepressants

28 during and after pregnancy to manage mood disorders, with possible implications for the developing

29 offspring. The microbiota within the gastrointestinal tract contributes to the regulation of serotonin

30 synthesis. However, the interaction between maternal depression, SSRI use, bacterial community

31 composition, and availability of microbiota-derived metabolites during pregnancy and lactation is not

32 clear and may be consequential to the long-term health of mother and offspring. To determine the impact

33 of SSRI treatment on maternal microbial community dynamics, we conducted these studies in a rat model

34 of maternal vulnerability (MV). All MV females are on a background of genetic vulnerability, where rats

35 exposed to early life stress (sMV) develop a depressive-like phenotype. In adulthood, sMV-and control

36 (cMV) females were treated with either the SSRI fluoxetine (FLX) or the vehicle (Veh) throughout

37 pregnancy and lactation. High-resolution 16S ribosomal RNA gene sequencing and targeted metabolomic

38 analysis were used to assess the fecal microbiome and metabolite availability, respectively.

39 Results: The diversity, structure, and composition of the fecal bacterial community differed between

40 pregnancy and lactation. Shifts in microbiota composition were accompanied by changes in fecal

41 metabolite availability. FLX altered some key features of the transition from pregnancy to lactation, but

42 only in females exposed to early life stress (sMV-Veh vs sMV-FLX). For instance, sMV-FLX females had

43 lower fecal availability of the amino acids serine, proline, and aspartic acid than sMV-Veh females. These

44 metabolite concentrations correlated negatively with the relative abundance of bacterial taxa Prevotella,

45 Ruminococcus, and Oscillospira.

46 Conclusions: Our studies demonstrate an important relationship between antidepressant use during the

47 perinatal period and maternal fecal metabolite availability in sMV rats, possibly through parallel changes

48 in the maternal gut microbiome. Since maternal microbial metabolites contribute to health outcomes in

49 offspring, insults to the maternal microbiome by SSRIs might have inter-generational consequences. 
51 Keywords: Fecal microbiome, fecal metabolome, pregnancy, lactation, depression, SSRI antidepressants,

52 fluoxetine, rat, serotonin transporter, 16s rRNA

\section{Background}

54 Dysregulation of the neurotransmitter serotonin in the brain is a widely recognized hallmark of major

55 depressive disorder $[1,2]$. Selective serotonin reuptake inhibitor (SSRI) antidepressants target the 56 serotonin transporter (SERT), thereby influencing the serotonergic tone [3]. Serotonin (also referred to as

57 5-hydroxytryptamine or 5-HT) and SERT are particularly well-known for playing crucial regulatory roles

58 in the brain, but they also act in many peripheral tissues. Nearly $95 \%$ of the body's serotonin resides

59 within the gastrointestinal tract, where it is mainly produced by enterochromaffin cells [4]. Interestingly,

60 germ-free and antibiotic-treated rodents show lower levels of colonic and serum serotonin. This is

61 reversible by microbial colonization, suggesting an important role for specific members of the gut

62 microbiome in modulating serotonin availability [5-11]. Indeed, microbiota synthesize tryptophan, the

63 precursor of serotonin, and promote serotonin biosynthesis in the host organism [8, 9]. Conversely, host

64 serotonin signaling impacts the gut microbiota composition, as we have shown using rats with reduced or

65 no SERT gene expression [12]. Several studies have found alterations in the gut microbiota composition

66 of patients with major depression versus healthy controls [13, 14]. The same has been observed in rodents

67 with depressive-like symptoms [15]. Moreover, a recent in vivo study showed that SSRIs modulate the gut

68 microbiota composition, an effect that may be related to in vitro antimicrobial properties of SSRIs [16-

$6920]$.

70 The maternal gut microbiota synthesizes a variety of metabolites that reach the systemic

71 circulation during pregnancy and lactation, and influence offspring innate immune development [21, 22].

72 Environmental and pharmacological insults disrupt the compositional and functional states of the maternal

73 gut microbiota, as recently demonstrated by several animal studies focusing on stress, diet, and antibiotic 
74 use during pregnancy [23-27]. Despite clear evidence that the microbiome interacts with serotonin

75 homeostasis, it is currently unknown whether alterations in serotonin homeostasis, such as those often co-

76 occurring with depression or resulting from SSRI use, affect the maternal gut microbiome during the

77 perinatal period [5, 8-12]. An estimated 7-13\% of women suffer from a major depressive disorder in the

78 perinatal period and $1-10 \%$ of pregnant women take SSRI antidepressants, further highlighting the

79 necessity to better understand the effect of SSRI medication during this period on the gut microbiota [28-

$8031]$.

We aimed to investigate whether a depressive-like phenotype, SSRI antidepressant treatment, and their combination affect the microbial community composition and function during pregnancy and the postpartum period. To this end, we used a rat model of maternal vulnerability (MV) in combination with early life stress by maternal separation (sMV) or control handling (cMV). In adulthood, sMV females show a lower sucrose preference and lower neural growth factor gene expression in the basolateral amygdala and paraventricular nucleus of the brain than cMV females [32]. Thus, sMV females show anhedonia, which is an endophenotype associated with depressive symptoms. We use sMV rats as a model for depression, allowing us to study the separate effects of the maternal depressive-like phenotype and SSRI use during pregnancy, as well as the combination of both.

In the current study, adult female sMV and cMV rats were treated daily with fluoxetine (FLX), a

91 commonly used SSRI, or vehicle (Veh) throughout pregnancy and lactation. There were 4 groups of MV

92 females; the control group that was treated with vehicle (cMV-Veh), the maternally separated group that

93 was treated with vehicle (sMV-Veh), the control group that was treated with fluoxetine (cMV-FLX), and

94 the maternally separated group that was treated with fluoxetine (sMV-FLX). Weekly fecal samples were

95 collected for 16S rRNA gene sequencing. In addition, targeted metabolomic analysis of amino acids,

96 short-chain fatty acids and bile acids was done on a subset of samples. We examined the hypothesis that

97 depressive-like symptoms and antidepressant treatment during pregnancy and lactation affect the maternal

98 fecal microbiome and its functional capacity. Specifically, we hypothesized that (1) pregnancy and

99 lactation have distinct fecal microbial signatures; (2) early life stress and FLX alter the fecal microbial 
100 signatures of pregnancy and lactation, and the combination of early life stress and FLX has the most

101 pronounced effect; (3) pregnancy and lactation have distinct fecal metabolic signatures; (4) early life

102 stress and FLX treatment alter the fecal metabolic signatures of pregnancy and lactation, and the 103 combination of early life stress and FLX has the most pronounced effect; and (5) the abundances of 104 microbial taxa correlate with the concentrations of fecal metabolites.

\section{Methods}

\section{Experimental animals}

107 The animals came from our colony of serotonin transporter knockout (SERT ${ }^{-/}$, Slc6a41 ${ }^{\mathrm{Hubr}}$ ) Wistar rats at 108 the University of Groningen (Groningen, the Netherlands), and were derived from outcrossing SERT ${ }^{+/-}$ 109 rats [33]. Animals were supplied ad libitum with standard lab chow (RMH-B, AB Diets; Woerden, the 110 Netherlands) and water and were kept on a 12:12 h light-dark cycle (lights off at 11:00 h), with an ambient 111 temperature of $21 \pm 2{ }^{\circ} \mathrm{C}$ and humidity of $50 \pm 5 \%$. Cages were cleaned weekly, and animals were 112 provided with a wooden stick for gnawing $(10 \times 2 \times 2 \mathrm{~cm})$ and nesting material (Enviro-driT ${ }^{\mathrm{TM}}$, Shepherd

113 Specialty Papers, Richland, MI, USA). During pregnancy, females were housed individually in type III 114 Makrolon cages (38.2x22.0x15.0cm) and stayed with their pups until postnatal day (PND)21. Animals 115 were weaned at PND21 and group housed in same-sex cages of 3-5 animals in type IV $116(55.6 \times 33.4 \times 19.5 \mathrm{~cm})$ Makrolon cages. Genotyping of the animals was performed as described previously 117 [12]. All experimental procedures were approved by the Institutional Animal Care and Use Committee of 118 The University of Groningen and were conducted in agreement with the Law on Animal Experiments of 119 The Netherlands.

\section{Early life stress by maternal separation in a rat model of maternal vulnerability}

121 Our rat model of maternal vulnerability (MV) consists of heterozygous SERT knockout (SERT ${ }^{+/}$) female 122 rats. MV rats show 40-50\% lower SERT gene expression levels than wildtypes [34]. These levels are 
123 similar to those of a considerable portion of the human population, who carry at least one short allele of

124 the SERT gene [35]. When human carriers of this allele experience severe stressful life events, they are at

125 higher risk of developing symptoms of depression [36]. We mimic this gene-by-environment interaction

126 contributing to depression in MV rats by exposing them to early life stress [37]. The early life stress

127 protocol was conducted as previously described [12]. In short, $\mathrm{SERT}^{+-}$females were mated with $\mathrm{SERT}^{+/-}$

128 males (F0). Females were left undisturbed until delivery, which was defined as PND0. From PND2 to

129 PND15, pups were either separated from the dam for 6 hours per day, or handled 15 minutes per day

130 (Figure 1a). The $\mathrm{SERT}^{+/-}$female pups from these nests (F1) then matured to become the sMV-and cMV

131 females.

\section{Breeding and fluoxetine treatment}

133 sMV and cMV females were mated with wildtype males. Females were between the age of 3 and 6

134 months, and mated when in estrus (checked with a disinfected impedance meter, model MK-11,

135 Muromachi, Tokyo, Japan). This was termed gestational day (GD)0. The males were removed after 24

136 hours, and the females stayed isolated in a type III cage. Throughout pregnancy and lactation, from GD1

137 until PND21, the dams were weighed and received an oral gavage of either $10 \mathrm{mg} / \mathrm{kg}$ fluoxetine (FLX,

138 Fluoxetine $20 \mathrm{PCH}$, Pharmachemie BV, the Netherlands) or vehicle daily at 11:00AM (Figure 1b).

139 Methylcellulose (MC; Sigma Aldrich Chemie BV, Zwijndrecht, the Netherlands) was used for vehicle

140 injections since it is the constituent of the fluoxetine capsule. FLX (5 mg/mL) and MC (1\%) solutions

141 were prepared with autoclaved water. The gavage volume ranged from $0.9 \mathrm{~mL}$ to $2.0 \mathrm{~mL}$ (depending on

142 body weight). Animals were treated orally by gently picking up the animal without restraint, and using

143 flexible PVC feeding tubes (40 cm length, Vygon, Valkenswaard, the Netherlands) in order to minimize

144 discomfort and stress in the animals. In total, 4 groups of MV females were sampled for this study: cMV-

145 Veh $(\mathrm{N}=20)$, sMV-Veh $(\mathrm{N}=13)$, cMV-FLX $(\mathrm{N}=34)$ and sMV-FLX $(\mathrm{N}=25)$. However, the final number of

146 animals selected for $16 \mathrm{~S}$ rRNA gene sequencing for this study were $11 \mathrm{cMV}-\mathrm{Veh}, 8 \mathrm{sMV}-\mathrm{Veh}, 7 \mathrm{cMV}-$ 
147 FLX and 6 sMV-FLX. Because littermates can share microbiotas, no females from the same litter were

148 used within each group.

\section{Fecal sample collection}

150 Fecal samples to be sequenced were collected at GD0 (before conception), GD7, GD14, PND2, PND7,

151 PND14 and PND21 (Figure 1b). We chose not to collect samples closer to the day of giving birth to not

152 induce unnecessary stress. The samples from GD7 and GD14 were grouped as pregnancy and the samples

153 from PND7, PND14 and PND21 as lactation, since we observed no significant differences within these

154 periods in terms of alpha diversity, beta diversity, and composition of the microbiome (data not shown), as

155 was also shown in a previous human study [38]. Conversely, the samples from PND2 were left out of the

156 analysis, since they did not fit either category (data not shown). The samples were freshly collected

157 directly from the animal between 10:00AM and 11:00AM, and precautions were taken to minimize

158 sample contamination, such as the use of gloves and disinfection of work surfaces. The samples were

159 placed in clean $2.0 \mathrm{~mL}$ Safe-Lock Eppendorf tubes (Nijmegen, The Netherlands), snap frozen in liquid

160 nitrogen, and stored at $-80^{\circ} \mathrm{C}$.

\section{$161 \quad$ 16S rRNA gene sequencing and analysis}

162 Genomic DNA was isolated from fecal pellets using the PSP® Spin Stool DNA Kit (STRATEC

163 Molecular GmbH, Berlin, Germany), according to the manufacturer's instructions for difficult to lyse

164 bacteria. About 100-200 mg fecal matter per sample was used for the DNA isolation; the remainder of the

165 sample was stored for metabolite measurements. A dual-index sequencing strategy targeting the V4 region

166 of the 16S rRNA gene was employed, using barcoded primers for the Illumina platform, as described

167 previously [39]. Library concentration was quantified using Qubit and an additional primer dimer clean-up

168 step was conducted using the AMPure XP beads according to the manufacturer's protocol (Beckman

169 Coulter, Brea, CA, USA). Sequencing was executed on a MiSeq instrument (Illumina, San Diego, CA)

170 using 250 base paired-end chemistry at the University of Pennsylvania Next Generation Sequencing Core.

171 For quality control purposes, a sample of the Human Microbiome Project Mock Community was included 
172 as a positive control, and water as a negative control. Quality filtering and chimera checking yielded

$1737,867,454$ quality-filtered sequences with a mean \pm SD depth of \pm 2560 reads per sample. $16 \mathrm{~S}$ rRNA gene

174 analysis was performed using mothur [40] and QIIME version 1.9.1 (Quantitative Insights Into Microbial

175 Ecology [41]) as described previously [23]. In short, Operational Taxonomic Units (OTUs) were defined

176 with $97 \%$ sequence similarity using clustering method CD-HIT. The samples were rarified to 1,000

177 sequences per sample before calculating diversity metrics. The Shannon diversity index was used to

178 calculate alpha diversity, and weighted UniFrac distances were used to calculate beta diversity.

\section{Statistical analysis}

180 To identify the fecal microbial OTUs that differentiate between pregnancy and lactation, the machine181 based learning algorithm Random Forests was used in R version 3.4.1 as previously described [23, 42182 44]. OTU importance was ranked by the percent increase in prediction error of the model as a result of 183 removal of that particular OTU from the model. The resulting model, derived from cMV-Veh samples, 184 was then applied to OTU tables from the other groups, to see how early life stress, FLX and their 185 combination alter the microbial signature of pregnancy and lactation. The R package "gplots" was used to plot OTU relative abundances as heat maps [45]. To predict the metabolic capacity of the gut microbiome

187 during pregnancy and lactation, a computational approach known as Phylogenetic Investigation of 188 Communities by Reconstruction of Unobserved States (PICRUSt [46]) was used. This algorithm uses the 189 16S rRNA data to predict the total metagenomic content, and these metagenomes are then mapped onto 190 functional Kyoto Encyclopedia of Genes and Genomes (KEGG) pathways in order to predict the full 191 functional capacity of the microbial community, as previously described [23]. Random Forests were 192 applied to the dataset of predicted metabolic pathways to identify those that shift from pregnancy to 193 lactation in the cMV-Veh group. Then, the impact of early life stress, FLX, and their combination on the 194 proportional representation of these predicted microbial metabolic pathways was plotted in a heat map. 


\section{Targeted metabolomics}

196 To draw associations between bacterial community composition, predicted gut microbial metabolic 197 capacity, and gut metabolite output, fecal metabolites were quantified in a subset of samples. GD7 was 198 chosen as a representative time point for pregnancy, and PND7 for lactation. Fecal samples weighing 199 about $300 \mathrm{mg}$ from 5 randomly selected animals per treatment group were used $(\mathrm{N}=36$ in total). Targeted 200 metabolomics was performed by the Microbial Culture and Metabolomics Core as part of the PennCHOP 201 Microbiome Program at the University of Pennsylvania via ultra-performance liquid chromatography 202 (Acquity UPLC system, Waters Corporation, Milford, MA). Amino acid concentrations were quantified with an AccQ-Tag Ultra C18 $1.7 \mu \mathrm{m} 2.1 \times 100 \mathrm{~mm}$ column and a photodiode array detector. Analysis was performed using the UPLC AAA H-Class Application Kit (Waters Corporation, Milford, MA) according to manufacturer's instructions. The limit of detection was $1 \mathrm{nmol} / \mathrm{g}$ stool. Bile acid concentrations were measured using a Cortecs UPLC C-18+1.6 mm 2.1 x $50 \mathrm{~mm}$ column, a QDa single quadrupole mass detector and an autosampler (192 sample capacity). The flow rate was $0.8 \mathrm{~mL} / \mathrm{min}$, the injection volume was $4 \mu \mathrm{L}$, the column temperature was $30^{\circ} \mathrm{C}$, the sample temperature was $4^{\circ} \mathrm{C}$, and the run time was 4 min per sample. Eluent A was $0.1 \%$ formic acid in water, eluent B was $0.1 \%$ formic acid in acetonitrile, the weak needle wash was $0.1 \%$ formic acid in water, the strong needle wash was $0.1 \%$ formic acid in acetonitrile, and the seal wash was $10 \%$ acetonitrile in water. The gradient was $70 \%$ eluent A for 2.5 minutes, gradient to $100 \%$ eluent B for 0.6 minutes, and then $70 \%$ eluent $\mathrm{A}$ for 0.9 minutes. The mass detection channels were: +357.35 for chenodeoxycholic acid and deoxycholic acid; +359.25 for lithocholic acid; +385.38 for obeticholic acid; +514.37 for glycoobeticholic acid; +528.39 for tauroobeticholic acid; -407.5 for cholic, alphamuricholic, betamuricholic, gamma muricholic, and omegamuricholic acids; -432.5 for glycolithocholic acid; -448.5 for glycochenodeoxycholic and

217 glycodeoxycholic acids; -464.5 for glycocholic acid; -482.5 for taurolithocholic acid; -498.5 for 218 taurochenodeoxycholic and taurodeoxycholic acids; and -514.4 for taurocholic acid. Samples were 219 quantified against standard curves of at least five points run in triplicate. The range of the assay was at 220 least $50 \mathrm{nM}-10,000 \mathrm{nM}$; the limit of detection was $<50 \mathrm{nM}$, and; the limit of quantitation was $>10,000$ 
nM. The limit of detection was $0.5 \mathrm{nmol} / \mathrm{g}$ stool. Finally, short-chain fatty acids were quantified using a

HSS T3 $1.8 \mu \mathrm{m} 2.1 \times 150 \mathrm{~mm}$ column with a photodiode array detector and an autosampler (192 sample acetonitrile in water. The gradient was $100 \%$ eluent A for $5 \mathrm{~min}$, gradient to $70 \%$ eluent B from 5-22 min,

$\mathrm{nm}$ resolution. Samples were quantified against standard curves of at least five points run in triplicate.

230 Concentrations in the samples were calculated as the measured concentration minus the internal standard.

231 The limit of detection was $1 \mathrm{nmol} / \mathrm{g}$ stool.

\section{Analysis of metabolomics data}

233 All metabolites that were detected in at least $40 \%$ of samples were included in the analysis. For these

234 metabolites, missing values were replaced by half the minimum positive value found for that metabolite,

235 assuming that missing values were the result of the concentration being below the detection limit. Random

236 Forests were applied as described above to the metabolite concentration table from the cMV-Veh samples

237 in order to reveal which individual metabolites were overrepresented during pregnancy or during lactation.

238 The resulting model was then applied to the data from the treatment groups, and heat maps were 239 generated. For predictive analysis, the open-access, online platform MetaboAnalyst 3.0 was used for 240 pathway analysis based on the metabolomics data [47]. Metabolite Set Enrichment Analysis, a 241 metabolomic version of Gene Set Enrichment Analysis, was used to investigate which sets of functionally 242 related metabolites differ between pregnancy and lactation per treatment group [48].

\section{Additional statistical methods}

244 Microbial alpha diversity was analyzed using a two-way ANOVA with period (pregnancy vs lactation) 245 and treatment group as between subject factors. Uncorrected Fisher's LSD was used for group 
comparisons within periods (cMV-Veh vs sMV-Veh, cMV-Veh vs cMV-FLX, cMV-Veh vs sMV-FLX, sMV-Veh vs sMV-FLX, cMV-FLX vs sMV-FLX within pregnancy and within lactation). Permutational multivariate analysis of variance using distance matrices (PERMANOVA) was used to analyze effects of pregnancy vs lactation and treatment group on weighted UniFrac distances [49]. PERMANOVA p-values were based on 999 Monte Carlo simulations. Multiple comparisons of OTU relative abundances and metabolite concentrations within pregnancy and within lactation between treatment groups were done by nonparametric Kruskal-Wallis tests followed by uncorrected Dunn's tests (cMV-Veh vs sMV-Veh, cMV-

Veh vs cMV-FLX, cMV-Veh vs sMV-FLX, sMV-Veh vs sMV-FLX, cMV-FLX vs sMV-FLX within pregnancy and within lactation). All of the OTUs and metabolites analyzed in this way differed between pregnancy and lactation in the cMV-Veh group, following from the Random Forests analysis; further statistics between periods were not conducted. A t-test was used to analyze the GD0 alpha diversity difference between cMV and sMV females. For the abovementioned tests, GraphPad Prism version 7

258 (GraphPad Software, Inc., San Diego, CA) was used. The statistical significance is indicated as follows:

${ }^{*} p<0.05 ; * * p<0.01$; and ${ }^{* * *} p<0.001, * * * * p<0.0001$. Data are presented as mean \pm SEM, except OTU relative abundance which is plotted as median \pm IQR.

\section{Results}

262 We examined the hypothesis that depressive-like symptoms and antidepressant treatment during 263 pregnancy and lactation affect the maternal fecal microbiome composition and function. Maternal 264 vulnerability (MV) rats were used, since early life stress produces depressive-like symptoms and shifts in 265 fecal microbiome community composition in these animals [12, 32]. In short, MV females exposed to 266 either maternal separation or control handling for the first 2 weeks of life were treated with fluoxetine or 267 vehicle daily throughout gestation and the postpartum period (Figure 1a,b). Thus, there were four groups 268 of MV females: control (cMV-Veh), maternally separated and vehicle-treated (sMS-Veh), fluoxetine- 
270 we have been confronted with a number of unexpected and thus far unexplained deaths of some MV

271 females as a result of fluoxetine treatment. About 25\% of FLX-treated females died, even though there

272 was no accumulation of fluoxetine or norfluoxetine levels in the blood over the course of treatment (data

273 not shown). However, the individual females used for the current study did not show any abnormal

274 response to FLX. Fecal pellets for 16s rRNA sequencing and analysis were collected during pregnancy at

275 gestational day (GD)7, GD14, and during lactation at postnatal day (PND)7, PND14 and PND21. A subset

276 of samples was used for targeted metabolomic analysis (Figure 1b).

\section{Pregnancy and lactation have distinct fecal microbial signatures}

278 To examine whether pregnancy and lactation have distinct fecal microbial signatures, we first analyzed the

279 16S rRNA sequencing data of pregnancy versus lactation in terms of microbial alpha diversity and

280 community structure over all 4 groups. Alpha diversity was higher during pregnancy than during lactation,

281 as shown by the Shannon diversity index (Figure 2a, $p<0.0001$ ). The differences in phylogenetic structure

282 between samples were explored by assessing weighted UniFrac distances. A separation between samples

283 obtained during pregnancy versus lactation was revealed by Principle Coordinates Analysis (PCoA)

284 (Figure 2b, PERMANOVA $p=0.001$ ).

285 In order to identify the bacterial signatures that underlie the observed shift in community structure

286 between pregnancy and lactation, a machine-learning approach called Random Forests was used to

287 analyze the data of the cMV-Veh group. This generated a model containing 28 Operational Taxonomic

288 Units (OTUs) at the genus-level, discriminating between pregnancy and lactation. A large reorganization

289 of the microbiome composition from gestation to lactation was revealed, consistent with structural

290 remodeling (Figure 2c; Supplemental Figure 1a). Particularly within the Bacteroidales and Clostridiales

291 orders, OTUs changed in their relative abundance from pregnancy to lactation (Figure 2c; Supplemental

292 Figure 1a). 


\section{Fluoxetine treatment alters the maternal fecal bacterial signatures of pregnancy and}

295 To test whether early life stress, FLX, and their combination alter the observed fecal microbial signatures

296 of pregnancy and lactation as observed in the cMV-Veh group, we first compared the groups with respect

297 to alpha- and beta diversity measures. We observed pre-conception differences between the sMV-and cMV group, with the sMV group showing higher alpha diversity than the cMV group (Supplemental

Figure $2 \mathrm{a}, p<0.05$ ), coinciding with differences in OTU relative abundance (Supplemental Figure $2 \mathrm{~b}$ ).

During pregnancy and lactation there was a similar pattern for the sMV-Veh but also the cMV-FLX and sMV-FLX groups; the mean Shannon diversity of the fecal microbial communities of the treatment groups PERMANOVA $p=0.001)$. In particular, microbial community structure in the sMV-FLX group differed from the structure in the sMV-Veh group, both during pregnancy and lactation (Figure 1d, pregnancy sMV-Veh vs sMV-FLX PERMANOVA $p<0.05 \mid$ lactation sMV-Veh vs sMV-FLX PERMANOVA $p=0.001)$. This suggested that, in addition to the dynamic restructuring of the fecal microbiome across pregnancy and lactation, fluoxetine treatment modulated community structure of the fecal microbiota in the sMV females during these periods.

Next, the relative abundance of the 28 OTUs constituting the Random Forests-generated model

314 discriminating between pregnancy and lactation in the cMV-Veh group was examined for the treatment

315 groups. Globally, the pattern of OTUs distinguishing between pregnancy and lactation in the treatment groups was similar to the cMV-Veh group (Figure 2c). However, specific OTUs showed variations in their 
with the largest contribution to the accuracy of the Random Forests model, Bacteroides and Prevotella

319 from the Bacteroidales order, were sensitive to FLX treatment. Specifically, both the cMV-FLX and 320 sMV-FLX had a lower relative abundance of Bacteroides than the cMV-Veh group during pregnancy, and 321 the sMV-FLX group had a lower relative abundance of Bacteroides than the sMV-Veh group during 322 lactation (Figure 2e, pregnancy cMV-Veh vs cMV-FLX $p<0.01$; vs sMV-FLX $p<0.05 \mid$ lactation sMV323 Veh vs sMV-FLX $p<0.05$ ). On the other hand, the sMV-FLX group had a higher relative abundance of 324 Prevotella than the sMV-Veh group during pregnancy and relative to the cMV-Veh, sMV-Veh and cMV325 FLX groups during lactation, reaching almost $25 \%$ of the total sample in lactation (Figure 2e, pregnancy sMV-Veh vs sMV-FLX $p<0.05 \mid$ lactation cMV-Veh vs sMV-FLX $p<0.05$, sMV-Veh vs sMV-FLX $p<0.01, \mathrm{cMV}-\mathrm{FLX}$ vs sMV-FLX $p<0.05$ ). Early life stress alone did not change the abundance of many OTUs relative to group that was control handled, although Ruminococcus was lower in the sMV-Veh group than in the cMV-Veh group during lactation (Figure 2e, lactation cMV-Veh vs sMV-Veh $p<0.05$ ).

This effect was reversed by FLX, as the sMV-FLX had a higher relative abundance than cMV-Veh and sMV-Veh during pregnancy, and a higher relative abundance than sMV-Veh during lactation (Figure 2e, pregnancy cMV-Veh/ sMV-Veh vs sMV-FLX $p<0.001$ | lactation sMV-Veh vs sMV-FLX $p<0.01$ ). we performed a correlation analysis to look at these relationships within our samples [50]. A correlation matrix between the 28 selected OTUs revealed that Prevotella was negatively correlated with a cluster consisting mainly of Clostridiales (Supplemental Figure 1d). Ruminoccus was the exception within the Clostridiales, being positively correlated with Prevotella (Supplemental Figure 1d).

Together, these results confirm that pregnancy and lactation are distinct with regard to fecal 339 microbiota diversity, structure, and composition. Early life stress increased alpha diversity during 340 lactation, as was the case pre-conception. There were no profound effects of early life stress alone on 341 either microbial community structure or on the relative abundance of OTUs resulting from our Random 342 Forests model. However, FLX impacted community structure during pregnancy and lactation, as well as 343 the relative abundance of a number of OTUs selected for their ability to distinguish between pregnancy 
344 and lactation. FLX in combination with early life stress had the largest effects on the maternal 345 microbiome.

\section{Pregnancy and lactation have distinct fecal metabolic signatures}

347 Bacterial communities metabolize and synthesize a number of metabolites that are essential for both dam 348 and offspring. Shifts in bacterial community composition and predictive function may suggest alterations 349 in the capacity of these communities to synthesize or metabolize these metabolites. We first performed 350 PICRUSt predictive analysis on the OTU data to explore the hypothesis that the functional potential of 351 pregnancy and lactation is different, since they have different microbial signatures. To determine which 352 distinct predictive metabolic pathways discriminate between pregnancy and lactation, Random Forests 353 analysis was performed on PICRUSt-generated data from the cMV-Veh group. Nine KEGG pathways 354 were significantly changed across pregnancy and lactation, including the pathway of lysine biosynthesis; 355 the pathway of alanine, aspartate, and glutamate metabolism; and the pathway of arginine and proline 356 metabolism (Figure 3a; Supplemental Figure 3a). Overall, pathways related to amino acid synthesis and 357 metabolism were among the metabolic pathways best able to characterize the difference in the predicted 358 function of the maternal bacterial community during pregnancy vs lactation.

To determine whether this altered predictive metabolic capacity of the microbiome was associated with actual metabolite availability, we measured the concentration of amino acids, bile acids, and shortchain fatty acids in a subset ( $\mathrm{N}=4-5$ per group in pregnancy and lactation) of fecal pellets. Corresponding to the microbiome results, principle component analysis (PCA) revealed a clear distinction in metabolic profile between samples obtained during pregnancy versus lactation (Figure 3b, upper graph).

To reveal the metabolic signatures of pregnancy and lactation, Random Forests analysis was 365 performed on the metabolite data from the cMV-Veh group. Fourteen metabolites were included in the 366 resulting model to distinguish between pregnancy and lactation (Supplemental Figure 3b). Most of them 367 were amino acids, and 11 out of 14 metabolites were higher in concentration during pregnancy than during 368 lactation (Figure 3c; Supplemental Figure 3c). For instance, the amino acids isoleucine and methionine 
had higher concentrations during pregnancy than during lactation, while proline concentrations were isovaleric acid concentrations decreased during lactation (Figure 3c; Supplemental Figure 3c). pathways that significantly differed between these two periods, again containing amino acid-related pathways such as glycine and serine metabolism (Figure 3e; Supplemental Figure 3e).

\section{Fluoxetine treatment alters maternal fecal metabolite availability during pregnancy}

\section{and lactation in a rat model for depressive-like behavior}

381 To investigate whether early life stress, FLX and their combination affect the abovementioned fecal

382 metabolic signatures of pregnancy and lactation as observed in the cMV-Veh group, we compared these 383 groups with respect to predicted- and measured metabolic data. The effects of early life stress and FLX on

384 the predictive pathways were mixed, with the sMV-FLX group having generally the lowest predicted 385 abundance of these metabolic pathways (Figure 3a). Visual inspection of the PCA graph based on the 386 metabolite concentration table revealed no clear effect of early life stress or FLX treatment (Figure 3b, 387 lower graph).

Next, the concentrations of the 14 metabolites included in the Random Forests-generated model 389 discriminating between pregnancy and lactation in the cMV-Veh group were examined for the treatment 390 groups. Similar to the microbiome results, the overall pattern of metabolite availability changes between 391 pregnancy and lactation was similar in all groups (Figure 3c). However, again, specific metabolites 392 showed variations in their concentration within pregnancy and/or lactation (Figure 3d; Supplemental 393 figure 3c). While there were no significant differences between the cMV-Veh and sMV-Veh group for any 
394 metabolites, the sMV-FLX group had low fecal concentrations of some amino acids (Figure 3d).

395 Concentrations of serine were lower in the sMV-FLX group than in the sMV-Veh group during 396 pregnancy, and for proline and aspartic acid this was the case during lactation (Figure 3d, serine 397 pregnancy sMV-Veh vs sMV-FLX $p<0.01$, proline lactation cMV-Veh/sMV-Veh vs sMV-FLX $p<0.05$, 398 aspartic acid lactation sMV-Veh vs sMV-FLX $p<0.05$ ). From the metabolites that were not selected by the 399 Random Forests model as distinguishing between pregnancy and lactation, the concentration of the bile 400 acids betamuricholic acid and omegamuricholic acid were decreased during pregnancy in the sMV-FLX 401 group compared to the cMV-Veh group and both the cMV-Veh- and the sMV-Veh group, respectively 402 (Supplemental Figure 3d, betamuricholic acid pregnancy cMV-Veh vs cMV-FLX/sMV-FLX $p<0.05$, 403 omegamuricholic acid pregnancy cMV-Veh/sMV-Veh vs sMV-FLX $p<0.05$ ). Similarly, the short-chain 404 fatty acid isobutyric acid was decreased in the sMV-FLX group relative to the Ctrl- and the sMV-Veh 405 group during lactation (Supplemental Figure 3d, cMV-Veh/sMV-Veh vs sMV-FLX $p<0.05$ ).

Then, we performed MSEA on the sMV-Veh, cMV-FLX and sMV-FLX data to determine which metabolites involved in specific metabolic processes were enriched in either pregnancy or lactation. The sMV-Veh group, like the cMV-Veh group, contained a number of metabolite sets that distinguished between pregnancy and lactation, such as methionine metabolism, and glycine and serine metabolism (Figure 3e; Supplemental Figure 3e). In contrast, in the cMV-FLX and sMV-FLX groups no metabolite abolished the differences in MSEA-identified pathways that distinguished the fecal metabolome during pregnancy from lactation as observed in the vehicle-treated groups. 


\section{The relative abundances of fecal bacterial taxa correlate with metabolite}

\section{concentrations}

421 Finally, we generated a correlation matrix to further explore the link between the variation in the 422 composition of the microbiome and variation in the availability of metabolites. A cluster consisting of 423 OTUs from the Clostridiales (including Oscillospira, Clostridia; other and UC Peptococcaceae) and 424 Desulfovibrionales (Desulfovibrio and UC Desulfovibrionaceae) orders was positively correlated with 425 methionine, and some of these OTUs also to other amino acids such as alanine, isoleucine glycine, lycine, 426 and threonine (Figure 3f). Showing almost the reverse pattern, the relative abundance of Prevotella was 427 negatively correlated with availability of isobutyric acid and a collection of amino acids; alanine, 428 isoleucine, glycine, methionine, and serine (Figure 3f). Associating to a different set of metabolites, the 429 relative abundance of Ruminococcus was inversely correlated with concentrations of aspartic acid and 430 some bile acids; betamuricholic acid, omegamuricholic acid, and alphamuricholic acid (Figure 3f). 431 Overall, the OTUs that were most affected in relative abundance by FLX treatment (Prevotella and 432 Ruminococcus, among others) are correlated with amino acid availability (serine, proline and aspartic 433 acid, among others).

\section{Discussion}

435 Our study shows that antidepressant treatment during pregnancy and lactation disrupts metabolite 436 availability in a rat model for maternal depressive-like symptoms, possibly through alterations in bacterial 437 community composition and function. In short, maternal vulnerability (MV) female rats were exposed to 438 early life stress (sMV) in the form of maternal separation. In adulthood, sMV and control (cMV) females

439 were treated throughout gestation and lactation with fluoxetine (FLX), a commonly prescribed selective 440 serotonin reuptake inhibitor (SSRI), or vehicle (Veh). Fecal samples were collected during pregnancy and 441 lactation, and 16s rRNA sequencing and targeted metabolomics were performed on these samples. 
443 females. We showed that the gut microbiome alpha diversity was relatively high during pregnancy, and

444 dropped during lactation. In addition, principal coordinates analysis revealed a shift in the structure of the

445 fecal microbiome from pregnancy to lactation. Using a machine-learning procedure called Random

446 Forests we identified the set of microbial taxa that was overrepresented in either pregnancy or lactation.

447 The resulting model, including 28 OTUs, characterized the shift in the maternal gut bacterial community

448 between pregnancy and lactation. Twenty-three OTUs in the resulting model were higher in abundance

449 during pregnancy than during lactation. In the literature there are several reports describing the changes in

450 the maternal microbiome over the course of gestation in humans and rodents $[23,38,51]$. We extend these

451 studies to define distinct microbial signatures associated with pregnancy and lactation.

452 Studies have shown that the mere presence of bacteria, but also the specific community 453 composition in the gut greatly affects plasma and fecal metabolite availability [52, 53]. To begin to 454 explore the hypothesis that, since pregnancy and lactation have different microbial signatures, their 455 functional capacity is also different, we performed PICRUSt predictive analysis on the OTU data. Using 456 Random Forests to determine the set of metabolic pathways that was best able to characterize the 457 difference between pregnancy and lactation, we identified a set of 9 KEGG pathways, a large proportion 458 of which was related to amino acid synthesis and metabolism. Gut microbes utilize metabolites as fuel, but 459 also modify and produce short-chain fatty acids, bile acids and amino acids that are used by the host [54, 460 55]. We measured the concentrations of these metabolites in a subset of fecal samples. Random Forests 461 analysis identified a set of 14 metabolites that best characterized the difference in fecal metabolite 462 availability between pregnancy and lactation in cMV-Veh rats. Most of these metabolites - 11 out of $14-$ 463 were higher in concentration during pregnancy than during lactation, reflecting either greater intake or 464 production of these metabolites during pregnancy, or more breakdown or transfer to the offspring during 465 lactation. Maternal (bacterial) metabolites have been shown to transfer to the developing offspring through 466 placental transfer and mother milk and play crucial roles in development $[22,56-58]$. We postulate that 
467 the observed shift in metabolic profile from pregnancy to lactation is an adaptive one, aiding the pregnant

468 and lactating female in providing nutrients and metabolites to the developing offspring.

469 We then hypothesized that early life stress and FLX alter the fecal microbial signatures of 470 pregnancy and lactation, and that the combination of early life stress and FLX produces the most 471 pronounced effect. An estimated 7-13\% of women are diagnosed with a major depressive disorder in the 472 perinatal period $[28,29]$. Numerous studies have identified differences between the gut microbiome of 473 depressed patients or depressive-like rodents, and healthy controls [13-15]. In this study, we used sMV 474 females as a rat model with a depressive-like phenotype [32]. It has been shown repeatedly that maternal 475 separation in rodents produces long-term effects not only on behavior, immune function and neurobiology, 476 but also on the gut microbiome [59-61]. Correspondingly, our lab showed previously that maternal 477 separation exacerbated the differences in the gut microbiome composition in young rats with a 478 heterozygous or complete knockout of SERT compared to wildtypes [12]. Consistent with these results, 479 we showed here that adult sMV females had a higher fecal alpha diversity than cMV females pre480 conception, during pregnancy and during lactation [32]. However, maternal separation did not affect the 481 structure and composition of the microbiome during pregnancy and lactation. It seems that the shift in maternal microbial community structure and composition from pregnancy to lactation is robust enough to withstand major perturbations by our early life stress protocol. In addition, no significant differences were found between the cMV-Veh and sMV-Veh groups in any metabolite concentration.

However, the SSRI fluoxetine was associated with differences in the maternal fecal microbiome and metabolic output in sMV rats. Around 1-10\% of women use SSRI antidepressants during pregnancy [30, 31]. Medication has the capacity to profoundly alter the gut microbiome composition [62, 63]. Indeed, there are several in vitro studies suggesting that SSRIs possess antimicrobial properties [16-19]. Moreover, it was shown recently that psychotropic drugs, including fluoxetine, are capable of altering the composition of the gut microbiome in rats [20]. Our results here confirm that SSRIs have the potential to modulate the fecal microbiome during pregnancy and lactation in rats. However, we only find significant 
493 Principle coordinates analysis showed an effect of FLX on the sMV bacterial community structure.

494 Similarly, when we compared the relative abundance of the Random Forests-identified taxa between the 495 treatments, most effects were seen in the sMV-FLX group relative to the sMV-Veh group. For instance, 496 fecal relative abundances of Prevotella and Ruminococcus were higher in the sMV-FLX group than in the 497 sMV-Veh group, at the cost of Bacteroides.

Thus, treatment with fluoxetine during pregnancy and lactation modulated the microbiome almost exclusively in the sMV animals, and not significantly in the cMV animals. There are several possible explanations for this. It might be that the sMV microbiome, belonging to a depressive-like host, is more sensitive to disruptions than the cMV microbiome, especially during the dynamic perinatal period. It might also be that both early life stress and FLX are "hits" that can shift the balance in the gut microbial community, and that under the current experimental conditions these "hits" are not large enough to be observed individually, but only when they are combined (a double-hit model). At the moment, there is a paucity of studies into the effect of SSRI on the microbiome from clinical studies on depression or animal studies mimicking this condition. For instance, it is unclear whether any effects SSRIs might have on the microbiome are part of their therapeutic potential or should rather be seen as side effects.

Then, we were interested in whether the effects of FLX on the maternal fecal microbiome would 
519 67]. During early pregnancy, maternal amino acids are only second to glucose in the amount of substrate

520 that crosses the placenta to support the growth of the developing fetus [68].

521 To further examine the metabolic pathways that were characteristic of either pregnancy or 522 lactation based on fecal metabolite concentrations and the effect of early life stress and FLX on these 523 pathways, metabolite set enrichment analysis was performed. Both the cMV-Veh and the sMV-Veh group 524 showed a clear signature of pregnancy vs lactation, reflected in a number of metabolic pathways 525 (including amino acid-related pathways) that were enriched in either period. Both the cMV-FLX and the 526 sMV-FLX group showed no such significantly enriched pathways, adding to the emerging picture that 527 FLX disrupts the fecal metabolic signatures of pregnancy and lactation. Future studies should address 528 whether this results in altered metabolite transfer to the offspring and what the possible consequences are 529 for offspring development.

Finally, to link the $16 \mathrm{~S}$ rRNA sequencing data with the metabolomic data, we generated correlation matrices both between the 28 OTUs selected by Random Forests analysis, as well as between

532 the relative abundance of these OTUs and metabolite concentrations. We found that the relative 533 abundance of Prevotella, enhanced in the sMV-FLX group, was negatively correlated with a cluster of 534 Clostridiales and Desulfovibrionales genera. Interestingly, these same Clostridiales and

535 Desulfovibrionales OTUs were positively associated with availability of amino acids. Previous literature 536 describes that some of the most prevalent amino acid fermenting microbes indeed belong to the 537 Clostridiales and Proteobacteria taxa [69]. The associations we report here yield a working hypothesis 538 that FLX - at least in an animal model for depressive-like behavior - tips the balance toward higher 539 abundance Prevotella and lower abundance of the aforementioned cluster of amino acid-fermenting 540 OTUs, which leads to lower levels of amino acids. Further mechanistic studies are needed to test this 541 hypothesis.

This study has several limitations. As with any 16S rRNA marker gene sequencing approach, our 543 analysis was constrained to the genus level. In addition, we are aware that the microbiome and its 544 functional capacity varies across locations in the gastrointestinal tract whereas we only used fecal samples 
545 [70]. However, our study design enabled us to sample longitudinally and to reduce the number of animals

546 needed to complete the study. Another issue is the observed mortality in about one-fourth of our SSRI-

547 treated females. This may be the result of the SERT ${ }^{+/-}$genotype of the MV females. Indeed, humans

548 carrying the short allele of the SERT gene polymorphism are more likely to suffer from side effects from

549 SSRIs than controls without a short allele [71]. In future studies, wildtype SERT ${ }^{+/+}$will have to be

550 included in order to establish whether this is indeed a genotype $\mathrm{X}$ drug effect. None of the females

551 included in the current study showed any signs of toxicity as a result of FLX. For future studies we also

552 suggest taking plasma samples, and possibly mother's milk, to confirm whether changes in fecal

553 metabolite concentrations correspond to systemic availability of these metabolites.

554 The benefits of performing microbiome research on laboratory rodents as opposed to humans

555 deserve mentioning here. For example, diet composition could be kept stable throughout the study,

556 whereas diet composition changes considerably from pre-conception to pregnancy in humans and even

557 short-term changes in diet are known to affect the gut microbiome $[72,73]$. In addition, we are able to

558 study the independent effect of SSRI use in rodents, as well as its interactions with a depressive-like

559 phenotype. Human SSRI-users always have underlying psychopathologies that are difficult to control for

560 [74]. However, the biggest asset of the current study is our ability to link changes to the microbiome and

561 predicted functional capacity of the bacterial community to changes in metabolite availability. Especially

562 in the context of pregnancy and lactation, any changes to maternal microbial metabolite availability might

563 have far-reaching consequences and are therefore important to assess in addition to the microbiome itself.

564 Our results add to the growing body of research linking serotonin signaling to the gut microbiome.

565 Maternal depression and SSRI use during pregnancy are both associated with detrimental developmental

566 outcomes in offspring. Clinical and animal studies have identified adverse effects such as lower birth

567 weight, delayed motor development, and increased anxiety [75]. Future studies will assess whether and to

568 what extent the maternal gut microbiome and metabolome might mediate these effects. Microbial

569 molecules have been shown to transfer from mother to offspring during gestation and through lactation to

570 support the development of the brain and the immune system, emphasizing their importance for offspring 
571 development [21, 22, 76, 77]. If the dampening effect of SSRI treatment on fecal amino acid

572 concentrations in a rodent model of depressive-like symptoms can be replicated in future studies, and if

573 these concentrations correlate with maternal and fetal plasma levels and to phenotypic outcomes in

574 offspring, dietary supplementation of amino acids alongside SSRI treatment during pregnancy and

575 lactation might be an important direction for future research.

\section{Conclusions}

577 We showed here that the fecal microbial and metabolic signatures of pregnancy and lactation in MV rats

578 are robust and are largely unaltered by early life stress with maternal separation. However, fluoxetine

579 treatment modulated key aspects of maternal microbial community dynamics and metabolite output in the

580 sMV females, including changes in the relative abundance of OTUs and associated decreases in amino

581 acid availability. This suggests that antidepressant use during pregnancy and lactation may lead to changes

582 in the microbiome. The current study adds to the emerging awareness that the microbiome is plastic and

583 vulnerable to environmental and pharmacological insults, especially during dynamic periods such as

584 pregnancy and lactation. We speculate that alterations to the maternal microbiome by SSRI treatment, and

585 the associated decreased availability of amino acids, reflect a compromised ability to supply nutrients to

586 the developing offspring.

\section{$587 \quad$ List of abbreviations}

588 cMV: maternal vulnerability females exposed to control handling; FLX: fluoxetine(-treated); IQR:

589 interquartile range; KEGG: Kyoto Encyclopedia of Genes and Genomes; L: lactation; MSEA: Metabolite

590 Set Enrichment Analysis; OTU: operational taxonomic unit; P: pregnancy; PCA: principal component

591 analysis; PC: principal component/coordinate; PCoA: principle coordinates analysis; PICRUSt:

592 Phylogenetic Investigation of Communities by Reconstruction of Unobserved States; SCFA: short-chain 
593 fatty acids; sMV: maternal vulnerability females exposed to early life stress; UC: unclassified; Veh:

594 vehicle-treated.

\section{Declarations}

\section{Ethics approval and consent to participate}

597 The animal study was approved by the Institutional Animal Care and Use Committee at the University of

598 Groningen (no. DEC-6936A), and was conducted in accordance with the institutional guidelines and the

599 Law on Animal Experiments.

\section{Consent for publication}

601 Not applicable.

\section{Competing interests}

603 The authors declare no competing interests.

\section{Funding}

605 This work was supported by the European Union's Horizon 2020 research and innovation program under

606 the Marie Sklodowska Curie Individual Fellowship (grant project no.: 660152-DEPREG) and NARSAD

607 young investigator grant (grant no.: 25206) awarded to JDAO. ASR was supported by a scholarship

608 awarded by the Fulbright Center The Netherlands.

\section{Authors' contributions}

610 ASR, EJ, TLB and JDAO designed the study. ASR and DJH performed the animal experiment and 611 collected the samples. ASR and EJ performed the wet lab work and bioinformatics analysis. ASR drafted 612 the manuscript. All authors contributed to critical revision of the manuscript. 


\section{Acknowledgements}

614 We thank Judith Swart, Wanda Douwenga and Christa Reitzema-Klein for their assistance with the early

615 life stress procedure, drug administration and sample collection.

\section{References}

617 1. Coppen A. The biochemistry of affective disorders. Br J Psychiatry. 1967;113:1237-64.

618 doi:10.1192/bjp.113.504.1237.

619 2. Fakhoury M. Revisiting the serotonin hypothesis: Implications for major depressive disorders. Mol

620 Neurobiol. 2016;53:2778-86. doi:10.1007/s12035-015-9152-z.

621 3. Claassen V, Davies JE, Hertting G, Placheta P. Fluvoxamine, a Specific 5-Hydroxytryptamine Uptake

622 Inhibitor. Br J Pharmacol. 1977;60:505-16.

623 4. Gershon MD, Tack J. The Serotonin Signaling System: From Basic Understanding To Drug

624 Development for Functional GI Disorders. Gastroenterology. 2007;132:397-414.

625 doi:10.1053/j.gastro.2006.11.002.

626 5. Sjögren K, Engdahl C, Henning P, Lerner UH, Tremaroli V, Lagerquist MK, et al. The gut microbiota 627 regulates bone mass in mice. J Bone Miner Res. 2012;27:1357-67. doi:10.1002/jbmr.1588.

628 6. Ge X, Ding C, Zhao W, Xu L, Tian H, Gong J, et al. Antibiotics - induced depletion of mice microbiota

629 induces changes in host serotonin biosynthesis and intestinal motility. J Transl Med. 2017;:1-9.

630 7. Hata T, Asano Y, Yoshihara K, Kimura-todani T, Miyata N, Zhang X, et al. Regulation of gut luminal 631 serotonin by commensal microbiota in mice. $2017 ;: 1-14$.

632 8. Reigstad CS, Salmonson CE, Rainey JF, Szurszewski JH, Linden DR, Sonnenburg JL, et al. Gut

633 microbes promote colonic serotonin production through an effect of short-chain fatty acids on

634 enterochromaffin cells. FASEB J. 2015;29:1395-403. doi:10.1096/fj.14-259598.

635 9. Yano JM, Yu K, Donaldson GP, Shastri GG, Ann P, Ma L, et al. Indigenous bacteria from the gut

636 microbiota regulate host serotonin biosynthesis. Cell. 2015;161:264-76. doi:10.1016/j.cell.2015.02.047. 
639 11. O’Mahony SM, Clarke G, Borre YE, Dinan TG, Cryan JF. Serotonin, tryptophan metabolism and the

640 brain-gut-microbiome axis. Behav Brain Res. 2015;277:32-48. doi:10.1016/j.bbr.2014.07.027.

641 12. El Aidy S, Ramsteijn AS, Dini-Andreote F, van Eijk R, Houwing DJ, Salles JF, et al. Serotonin

642 transporter genotype modulates the gut microbiota composition in young rats, an effect augmented by

643 early life stress. Front Cell Neurosci. 2017;11 August:1-12. doi:10.3389/fncel.2017.00222.

644 13. Naseribafrouei A, Hestad K, Avershina E, Sekelja M, Linløkken A, Wilson R, et al. Correlation

645 between the human fecal microbiota and depression. Neurogastroenterol Motil. 2014;26:1155-62.

646 14. Jiang H, Ling Z, Zhang Y, Mao H, Ma Z, Yin Y, et al. Altered fecal microbiota composition in

647 patients with major depressive disorder. Brain Behav Immun. 2015;48:186-94.

648 doi:10.1016/j.bbi.2015.03.016.

649 15. Park AJ, Collins J, Blennerhassett PA, Ghia JE, Verdu EF, Bercik P, et al. Altered colonic function 650 and microbiota profile in a mouse model of chronic depression. Neurogastroenterol Motil. 2013;25.

651 16. Muñoz-Bellido JL, Muñoz-Criado S, García-Rodríguez JA. In-vitro activity of psychiatric drugs

652 against Corynebacterium urealyticum (Corynebacterium group D2). J Antimicrob Chemother.

$6531996 ; 37: 1005-9$.

654 17. Bohnert JA, Szymaniak-Vits M, Schuster S, Kern W V. Efflux inhibition by selective serotonin 655 reuptake inhibitors in Escherichia coli. J Antimicrob Chemother. 2011;66:2057-60.

656 18. Macedo D, Filho AJMC, Soares de Sousa CN, Quevedo J, Barichello T, Júnior HVN, et al.

657 Antidepressants, antimicrobials or both? Gut microbiota dysbiosis in depression and possible implications

658 of the antimicrobial effects of antidepressant drugs for antidepressant effectiveness. J Affect Disord.

659 2017;208 September 2016:22-32. doi:10.1016/j.jad.2016.09.012.

660 19. Evrensel A, Ceylan ME. Gut-Microbiota-Brain Axis and Depression. In: Kim Y-K, editor.

661 Understanding Depression. Singapore: Springer Singapore; 2018. p. 197-207. doi:10.1007/978-981-10-

662 6580-4_17. 

(Berl). 2018;:1-15. doi:10.1007/s00213-018-5006-5. influences blood-brain barrier permeability in mice. Sci Transl Med. 2014;6:263ra158-263ra158. doi:10.1126/scitranslmed.3009759. microbiota drives early postnatal innate immune development. Science (80- ). 2016;351:1296-302. doi:10.1126/science.aad2571.

23. Jašarević E, Howard CD, Misic AM, Beiting DP, Bale TL. Stress during pregnancy alters temporal and spatial dynamics of the maternal and offspring microbiome in a sex-specific manner. Sci Rep. 2017;7 March:44182. doi:10.1038/srep44182. Microbes. 2015;0976 January 2016:310-20. 
689 regression of the prevalence and incidence of perinatal depression. J Affect Disord. 2017;219 May:86-92.

690 doi:10.1016/j.jad.2017.05.003.

691 30. El Marroun H, Jaddoe VW V., Hudziak JJ, Roza SJ, Steegers EAP, Hofman A, et al. Maternal use of

692 selective serotonin reuptake inhibitors, fetal growth, and risk of adverse birth outcomes. Arch Gen

693 Psychiatry. 2012;69:706-14. doi:10.1001/archgenpsychiatry.2011.2333.

694 31. Cooper WO, Willy ME, Pont SJ, Ray WA. Increasing use of antidepressants in pregnancy. Am J

695 Obstet Gynecol. 2007;196:544.e1-5.

696 32. Houwing DJ, Ramsteijn AS, Riemersma IW, Olivier JDA. Maternal separation induces anhedonia in

697 female heterozygous serotonin transporter knockout rats. Manuscr Submitt Publ. 2018.

698 33. Smits BMG, Mudde JB, van de Belt J, Verheul M, Olivier J, Homberg J, et al. Generation of gene

699 knockouts and mutant models in the laboratory rat by ENU-driven target-selected mutagenesis.

700 Pharmacogenet Genomics. 2006;16:159-69.

701 34. Homberg JR, Olivier JDA, Smits BMG, Mul JD, Mudde J, Verheul M, et al. Characterization of the

702 serotonin transporter knockout rat: A selective change in the functioning of the serotonergic system.

703 Neuroscience. 2007;146:1662-76.

704 35. Haberstick BC, Smolen A, Williams RB, Bishop GD, Foshee VA, Thornberry TP, et al. Population

705 Frequencies of the Triallelic 5HTTLPR in Six Ethnicially Diverse Samples from North America,

706 Southeast Asia, and Africa. Calcif Tissue Int. 2015;96:255-61.

707 36. Caspi A, Sugden K, Moffitt TE, Taylor A, Craig IW, Harrington H, et al. Influence of life stress on

708 depression: Moderation by a polymorphism in the 5-HTT gene. Science. 2003;301:386-9.

709 37. Houwing DJ, Buwalda B, van der Zee EA, de Boer SF, Olivier JDA. The Serotonin Transporter and

710 Early Life Stress: Translational Perspectives. Front Cell Neurosci. 2017;11 April:1-16.

711 doi:10.3389/fncel.2017.00117.

712 38. DiGiulio DB, Callahan BJ, McMurdie PJ, Costello EK, Lyell DJ, Robaczewska A, et al. Temporal and

713 spatial variation of the human microbiota during pregnancy. Proc Natl Acad Sci. 2015;112:11060-5.

714 doi:10.1073/pnas.1502875112. 

sequencing platform. Appl Environ Microbiol. 2013;79:5112-20. microbial communities. Appl Environ Microbiol. 2009;75:7537-41. analysis of high-throughput community sequencing data. Nat Methods. 2010;7:335-6. doi:10.1038/nmeth.f.303.

42. R: A language and environment for computing. 2017. https://www.r-project.org/.

43. Liaw a, Wiener M. Classification and Regression by randomForest. R news. 2002;2 December:18-22. doi:10.1038/nature13421.

45. Warnes GR, Bolker B, Bonebakker, Lodewijk Gentleman R, Huber W, Liaw A, Lumley T, et al. gplots: Various R programming tools for plotting data. 2016. https://cran.r-project.org/package=gplots.

46. Langille MGI, Zaneveld J, Caporaso JG, McDonald D, Knights D, Reyes JA, et al. Predictive 
9993.2001.01070.pp.x.

742 50. Faust K, Sathirapongsasuti JF, Izard J, Segata N, Gevers D, Raes J, et al. Microbial co-occurrence

743 relationships in the Human Microbiome. PLoS Comput Biol. 2012;8.

744 51. Koren O, Goodrich JK, Cullender TC, Spor A, Laitinen K, Kling Bäckhed H, et al. Host remodeling of

745 the gut microbiome and metabolic changes during pregnancy. Cell. 2012;150:470-80.

746 52. Wikoff WR, Anfora AT, Liu J, Schultz PG, Lesley S a, Peters EC, et al. Metabolomics analysis

747 reveals large effects of gut microflora on mammalian blood metabolites. Proc Natl Acad Sci U S A.

$748 \quad 2009 ; 106: 3698-703$. doi:10.1073/pnas.0812874106.

749 53. Marcobal A, Kashyap PC, Nelson TA, Aronov PA, Donia MS, Spormann A, et al. A metabolomic

750 view of how the human gut microbiota impacts the host metabolome using humanized and gnotobiotic

751 mice. ISME J. 2013;7:1933-43. doi:10.1038/ismej.2013.89.

752 54. Lin R, Liu W, Piao M, Zhu H. A review of the relationship between the gut microbiota and amino acid

753 metabolism. Amino Acids. 2017;49:2083-90.

754 55. Nieuwdorp M, Gilijamse PW, Pai N, Kaplan LM. Role of the microbiome in energy regulation and

755 metabolism. Gastroenterology. 2014;146:1525-33. doi:10.1053/j.gastro.2014.02.008.

756 56. van de Pavert SA, Ferreira M, Domingues RG, Ribeiro H, Molenaar R, Moreira-Santos L, et al.

757 Maternal retinoids control type 3 innate lymphoid cells and set the offspring immunity. Nature.

$758 \quad 2014 ; 508: 123-7$. doi:10.1038/nature13158.

759 57. Thorburn AN, McKenzie CI, Shen S, Stanley D, MacIa L, Mason LJ, et al. Evidence that asthma is a

760 developmental origin disease influenced by maternal diet and bacterial metabolites. Nat Commun. 2015;6.

761 58. Scholtens DM, Bain JR, Reisetter AC, Muehlbauer MJ, Nodzenski M, Stevens RD, et al. Metabolic

762 networks and metabolites underlie associations between maternal glucose during pregnancy and newborn

763 size at birth. Diabetes. 2016;65:2039-50.

764 59. Bailey MT, Coe CL. Maternal separation disrupts the integrity of the intestinal microflora in infant

765 rhesus monkeys. Dev Psychobiol. 1999;35:146-55.

766 60. De Palma G, Blennerhassett P, Lu J, Deng Y, Park a. J, Green W, et al. Microbiota and host 
767 determinants of behavioural phenotype in maternally separated mice. Nat Commun. 2015;6 August:7735.

768 doi:10.1038/ncomms8735.

769 61. Pusceddu MM, El Aidy S, Crispie F, O’Sullivan O, Cotter P, Stanton C, et al. N-3 polyunsaturated

770 fatty acids (PUFAs) reverse the impact of early-life stress on the gut microbiota. PLoS One. 2015;10:1-

$771 \quad 13$.

772 62. Devkota S. Prescription drugs obscure microbiome analyses. Science (80- ). 2016;351:452-3.

773 63. Falony G, Joossens M, Vieira-Silva S, Wang J, Darzi Y, Faust K, et al. Population-level analysis of

774 gut microbiome variation. Science (80- ). 2016;352:560-4.

775 64. Zhao L, Xiong Z, Lu X, Zheng S, Wang F, Ge L, et al. Metabonomic evaluation of chronic

776 unpredictable mild stress-induced changes in rats by intervention of fluoxetine by HILIC-UHPLC/MS.

777 PLoS One. 2015;10:1-14.

778 65. Metges CC. Contribution of microbial amino acids to amino acid homeostasis of the host. J Nutr.

779 2000;130:1857S-64S. doi:10.1093/jn/130.7.1857S.

780 66. Wu G, Wu Z, Dai Z, Yang Y, Wang W, Liu C, et al. Dietary requirements of "nutritionally non-

781 essential amino acids" by animals and humans. Amino Acids. 2013;44:1107-13.

782 67. Lin G, Wang X, Wu G, Feng C, Zhou H, Li D, et al. Improving amino acid nutrition to prevent

783 intrauterine growth restriction in mammals. Amino Acids. 2014;46:1605-23.

784 68. Herrera E. Metabolic adaptations in pregnancy and their implications for the availability of substrates

785 to the fetus. Eur J Clin Nutr. 2000;54:S47-51.

786 69. Dai Z-L, Wu G, Zhu W-Y. Amino acid metabolism in intestinal bacteria: links between gut ecology

787 and host health. Front Biosci (Landmark Ed. 2011;16 September:1768-86.

788 http://www.ncbi.nlm.nih.gov/pubmed/21196263.

789 70. Li D, Chen H, Mao B, Yang Q, Zhao J, Gu Z, et al. Microbial Biogeography and Core Microbiota of

790 the Rat Digestive Tract. Sci Rep. 2017;8 March:1-16. doi:10.1038/srep45840.

791 71. Luddington NS, Mandadapu A, Husk M, El-Mallakh RS. Clinical Implications of Genetic Variation in

792 the Serotonin Transporter Promoter Region. Prim Care Companion J Clin Psychiatry. 2009;11:93-102. 
doi:10.4088/PCC.08r00656.

794 72. Hillier SE, Olander EK. Women's dietary changes before and during pregnancy: A systematic review.

795 Midwifery. 2017;49 June 2016:19-31.

796 73. David LA, Maurice CF, Carmody RN, Gootenberg DB, Button JE, Wolfe BE, et al. Diet rapidly and

797 reproducibly alters the human gut microbiome. Nature. 2014;505:559-63. doi:10.1038/nature12820.

798 74. Olivier JDA, Åkerud H, Sundström Poromaa I. Antenatal depression and antidepressants during

799 pregnancy: Unraveling the complex interactions for the offspring. Eur J Pharmacol. 2015;753:257-62.

800 doi:10.1016/j.ejphar.2014.07.049.

801 75. Olivier JDA, Akerud H, Kaihola H, Pawluski JL, Skalkidou A, Högberg U, et al. The effects of

802 maternal depression and maternal selective serotonin reuptake inhibitor exposure on offspring. Front Cell

803 Neurosci. 2013;7 May:73. doi:10.3389/fncel.2013.00073.

804 76. Thion MS, Low D, Silvin A, Chen J, Grisel P, Schulte-Schrepping J, et al. Microbiome influences

805 prenatal and adult microglia in a sex-specific manner. Cell. 2017;:1-17. doi:10.1016/j.cell.2017.11.042.

806 77. Jašarević E, Howard CD, Morrison K, Misic A, Weinkopff T, Scott P, et al. The maternal vaginal

807 microbiome partially mediates the effects of prenatal stress on offspring gut and hypothalamus. Nat

808 Neurosci. 2018. doi:10.1038/s41593-018-0182-5.

809

810

811

812

813

814 


\section{$815 \quad$ Figure legends}

816 Figure 1: Overview of study design and sampling schedule.

817 (a) Early life stress protocol. $\mathrm{SERT}^{+/-}$females were crossed with $\mathrm{SERT}^{+/-}$males, yielding nests with 818 offspring genotypes $\mathrm{SERT}^{+/}, \mathrm{SERT}^{+/}$, and $\mathrm{SERT}^{-/}$. The $\mathrm{SERT}^{+/-}$females are our model of maternal 819 vulnerability (MV). From postnatal day (PND) 2 to PND15, pups were either maternally separated for 6 820 hours per day (early life stress - sMV) or control handled (cMV) for 15 minutes per day. Pups were 821 weaned at PND21. sMV- and cMV females were group housed (same treatment) until adulthood. (b)

822 Fluoxetine treatment during pregnancy and lactation, and fecal sampling schedule. Adult sMV and 823 cMV females ( $\mathrm{N}=14-18$ /group) were crossed with wildtype males. Through pregnancy and lactation, from 824 gestational day (GD)1 until PND21, females received a daily oral injection of either $10 \mathrm{mg} / \mathrm{kg}$ fluoxetine 825 (FLX) or methylcellulose (MC, Veh). Thus, there were 4 groups of females: cMV-Veh, sMV-Veh, cMV826 FLX, and sMV-FLX (N=6-11/ group). Fecal pellets for 16s rRNA gene sequencing were freshly collected 827 before conception at GD0, during pregnancy at GD7 and GD14, at PND2, and during lactation at PND7, 828 PND14 and PND21 (N=192 in total). Selected fecal samples from GD7 and PND7 were also used for metabolomic analysis $(\mathrm{N}=4-5 /$ group per time point, $\mathrm{N}=36$ in total).

Figure 2: Fluoxetine treatment alters the microbiome during pregnancy and lactation in rats with a measure of alpha diversity. A two-way ANOVA was performed with period (pregnancy vs lactation) and group as factors. Uncorrected Fisher's LSD was used for group comparisons within periods. (b) Structure of the microbial communities during pregnancy and lactation. Communities were clustered using PCoA of the weighted UniFrac distance matrix. Each point corresponds to the microbial community of one sample that was collected during pregnancy or lactation. The percentage of variation explained by the PC is indicated on the axes. Colors correspond to period; all samples were grouped within pregnancy and

838 lactation. (c) Heatmap of relative abundances of Random Forests-identified OTUs distinguishing 839 between pregnancy and lactation. Random Forests was used to identify the microbial OTUs that had the 
840 highest predictive value for distinguishing between pregnancy and lactation in the cMV-Veh group. The

841 heatmap depicts the relative abundance of these 28 OTUs during pregnancy and lactation for all groups,

842 ordered by unsupervised clustering. (d) The effect of FLX on the structure of the sMV microbiome

843 during pregnancy and lactation. Communities were clustered using PCoA of the weighted UniFrac

844 distance matrix. Each point corresponds to the microbial community of one sample that was collected

845 during pregnancy (upper graph) or lactation (lower graph). The percentage of variation explained by the

846 PC is indicated on the axes. Colors correspond to group; sMV-Veh in white and sMV-FLX in grey. (e)

847 Relative abundance of selected Random Forests-identified OTUs. Kruskal-Wallis tests were used, with

848 subsequent group comparisons within periods (see Methods). N=12-22 samples/group from pregnancy

$849(\mathrm{~N}=64$ in total), $\mathrm{N}=18-33$ /group from lactation $(\mathrm{N}=96$ in total).

$850 \quad$ Figure 3: Fluoxetine alters fecal metabolite availability during pregnancy and lactation in rats with 851 a depressive-like phenotype. (a) Heatmap of Random Forests-identified PICRUSt-generated KEGG

852 pathways distinguishing between pregnancy and lactation. Z-scores are depicted of the abundance of

853 the 9 identified pathways for all groups. The pathways are ordered by unsupervised clustering. (b)

854 Structure of the metabolomic composition during pregnancy and lactation. Samples were clustered

855 using PCA of the metabolomics concentration table. Each point corresponds to the metabolic capacity of

856 one sample that was collected during pregnancy or lactation. The percentage of variation explained by the

857 PC is indicated on the axes. Colors correspond to period in the upper graph, and to group in the lower

858 graph. (c) Heatmap of Random Forests-identified metabolite concentrations distinguishing between

859 pregnancy and lactation. Z-scores are depicted of the concentrations of the 14 identified metabolites for

860 all groups. (d) Concentrations of selected amino acids. Kruskal-Wallis tests were used, with subsequent

861 group comparisons within periods (see Methods). (e) Metabolite Set Enrichment Analysis of pregnancy

862 vs lactation per group. Only the pathways that were significantly altered between pregnancy and

863 lactation are shown here; the full list of metabolic pathways is shown in Supplemental Figure 3e. (f)

864 Correlation matrix of metabolites and OTUs throughout pregnancy and lactation. Spearman's rank

865 correlations were determined between the 14 Random Forests-identified metabolites and the 28 Random 
Forests-identified OTUs. The circles represent the correlation between each metabolite and OTU. The size

867 of the circle increases with decreasing $p$-value-, and the color of the circle corresponds to the $\rho$-value of

868 the correlation (the strength of the correlation). Only significant $(p<0.05)$ correlations are plotted, and only

869 the OTUs with at least 1 significant correlation are shown here. The matrix is ordered by hierarchical

870 clustering. For a: $\mathrm{N}=12-22$ samples/group from pregnancy ( $\mathrm{N}=64$ in total), $\mathrm{N}=18-33$ /group from lactation

871 ( $\mathrm{N}=96$ in total). For b-f: $\mathrm{N}=4-5 /$ group from pregnancy $(\mathrm{N}=18$ in total), $\mathrm{N}=4-5 /$ group from lactation $(\mathrm{N}=18$

872 in total).

873 Supplemental Figure 1: The effect of early life stress and fluoxetine on the microbiome during 874 pregnancy and lactation. (a) Random Forests model to determine which OTUs were best able to 875 distinguish between pregnancy and lactation in the cMV-Veh group. The inset figure shows 876 increasing numbers of $97 \%$-identity OTUs on the $\mathrm{x}$-axis plotted against the cross-validation error of the 877 model on the y-axis. It was determined that the optimal model would include the top 28 OTUs, which are 878 shown in the bigger bar graph. From top to bottom, the OTUs are shown in descending order, 879 corresponding to their importance to discriminating between pregnancy and lactation. The length of the bars represents the mean decrease in accuracy of the model if this OTU would be removed from the model. (b) Structure of the microbial communities during pregnancy and lactation. Communities were clustered using PCoA of the weighted UniFrac distance matrix. Each point corresponds to the microbial community of one sample that was collected during pregnancy or lactation. The percentage of variation explained by the PC is indicated on the axes. Colors correspond to group (containing samples from both pregnancy and lactation). (c) Bar graphs showing relative abundance of selected Random

Forests-identified OTUs. A Kruskal-Wallis test was used, with subsequent group comparisons within periods (see Methods). Only OTUs with a median relative abundance $>0$ in at least 1 of the groups are shown here. (d) Correlation matrix of OTUs throughout pregnancy and lactation. Spearman's rank correlations were determined between the 28 Random Forests-identified OTUs. The circles represent the correlation between two given OTUs, with the size of the circle increasing with decreasing $p$-value of the 
correlation). Only significant $(p<0.05)$ correlations are plotted. The OTUs are ordered by hierarchical

893 clustering. $\mathrm{N}=12-22$ samples/group from pregnancy $(\mathrm{N}=64$ in total), $\mathrm{N}=18-33$ /group from lactation $(\mathrm{N}=96$

894 in total).

895 Supplemental Figure 2: The effect of early life stress on the gut microbiome in adult MV female

rats. a) Pre-conception alpha diversity. A t-test was used to test whether adult cMV and sMV females

differed in terms of fecal microbial Shannon Diversity. (b) Pre-conception microbiome composition. A

stack plot of the 20 most abundant OTUs was used to compare the fecal microbiome composition of cMV and sMV females. $\mathrm{N}=18$ for $\mathrm{cMV} ; \mathrm{N}=14$ for $\mathrm{sMV}$.

900 Supplemental Figure 3: The effect of early life stress and fluoxetine on metabolite availability

during pregnancy and lactation. (a) Random Forests model to determine which predicted KEGG

metabolic pathways were best able to distinguish between pregnancy and lactation in the cMV-Veh

group. The inset figure shows increasing numbers of KEGG metabolic pathways on the $\mathrm{x}$-axis plotted

against the cross-validation error of the model on the y-axis. It was determined that the optimal model

would include the top 9 KEGG metabolic pathways. The length of the bars represents the mean decrease

in accuracy of the model if this KEGG pathway would be removed from the model. (b) Random Forests

model to determine which metabolites were best able to distinguish between pregnancy and

lactation in the cMV-Veh group. The smaller figure shows increasing numbers metabolites on the $\mathrm{x}$-axis

bars represents the mean decrease in accuracy of the model if this metabolite would be removed from the 
918 a: $\mathrm{N}=22 \mathrm{cMV}-$ Veh samples from pregnancy and $\mathrm{N}=33$ from lactation. For $\mathrm{b}: \mathrm{N}=5 \mathrm{cMV}-\mathrm{Veh}$ samples from

919 pregnancy and $\mathrm{N}=5$ from lactation. For c: $\mathrm{N}=4-5$ /group from pregnancy and $\mathrm{N}=4-5 /$ group from lactation

$920 \quad(\mathrm{~N}=36$ in total $)$.

921 
a

Maternal separation $6 \mathrm{hrs} /$ day 4

q SMV

bioRxiv preprint doi: https://doi.org/10.1101 5501742 ; this version posted December 19, 2018. The copyright holder for this preprint (which was a

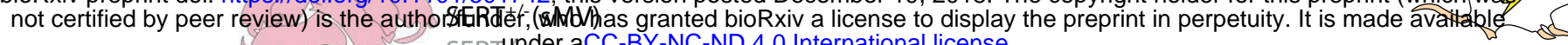
SERT Under aCC-BY-NC-ND 4.0 International license.

Control handling $15 \mathrm{~min} /$ day

SERT +1

SERT ${ }^{+/-}(\mathrm{CMV})$

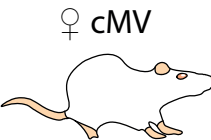

Birth

SERT ${ }^{-1}$

Weaning

Adulthood

Maternal separation or control handling PND2-PND15

b

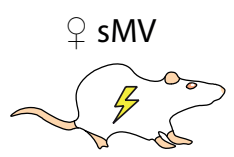

Pregnancy

$\mathrm{CMV}$

GD0

\begin{tabular}{lr}
\multicolumn{2}{c}{ Pregnancy } \\
\hline GD7 GD14
\end{tabular}

Daily oral gavage of $10 \mathrm{mg} / \mathrm{kg}$ fluoxetine (FLX) or vehicle (Veh)

Key

Fecal sample for $16 s$ rRNA sequencing

- Fecal sample for 16 s rRNA sequencing and metabolomics

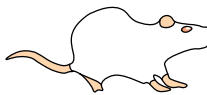

(-) 

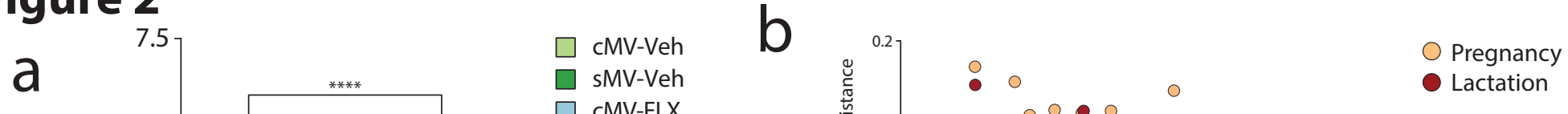

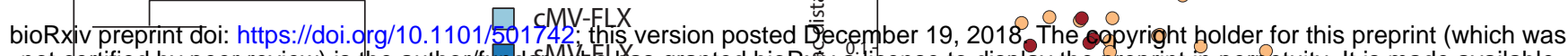

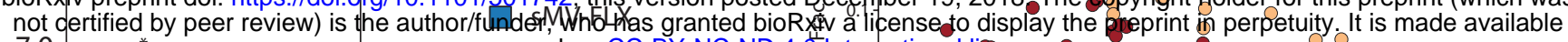

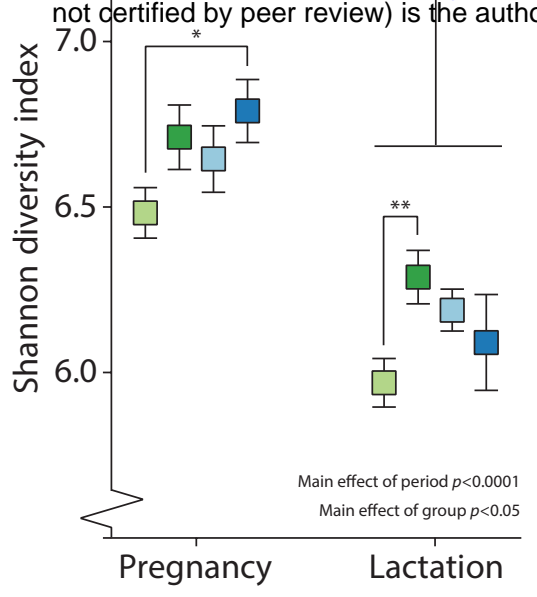

under aCC-BY-NC-ND 4.0EInternational license.

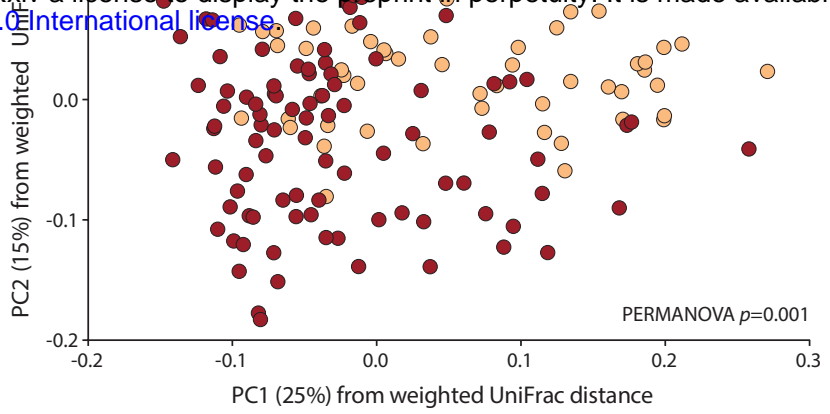

d

\section{Pregnancy}

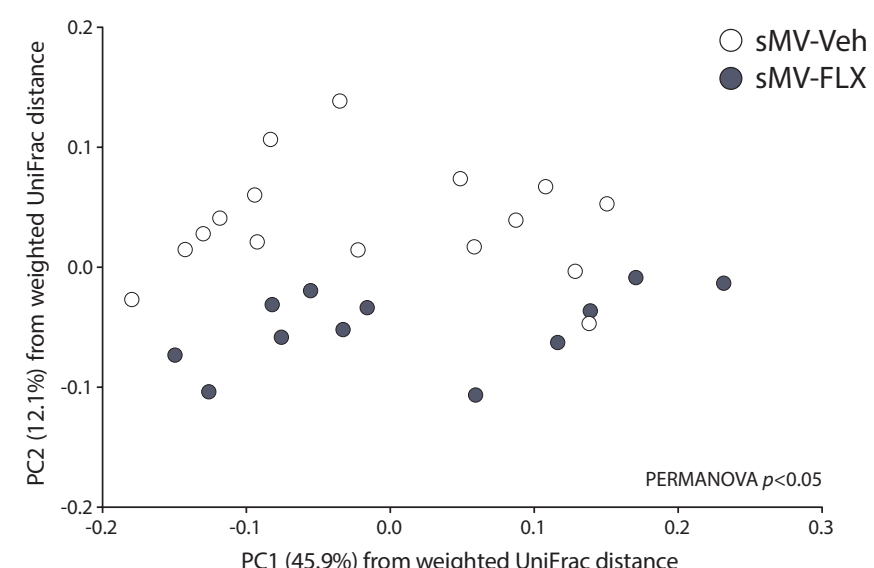

C

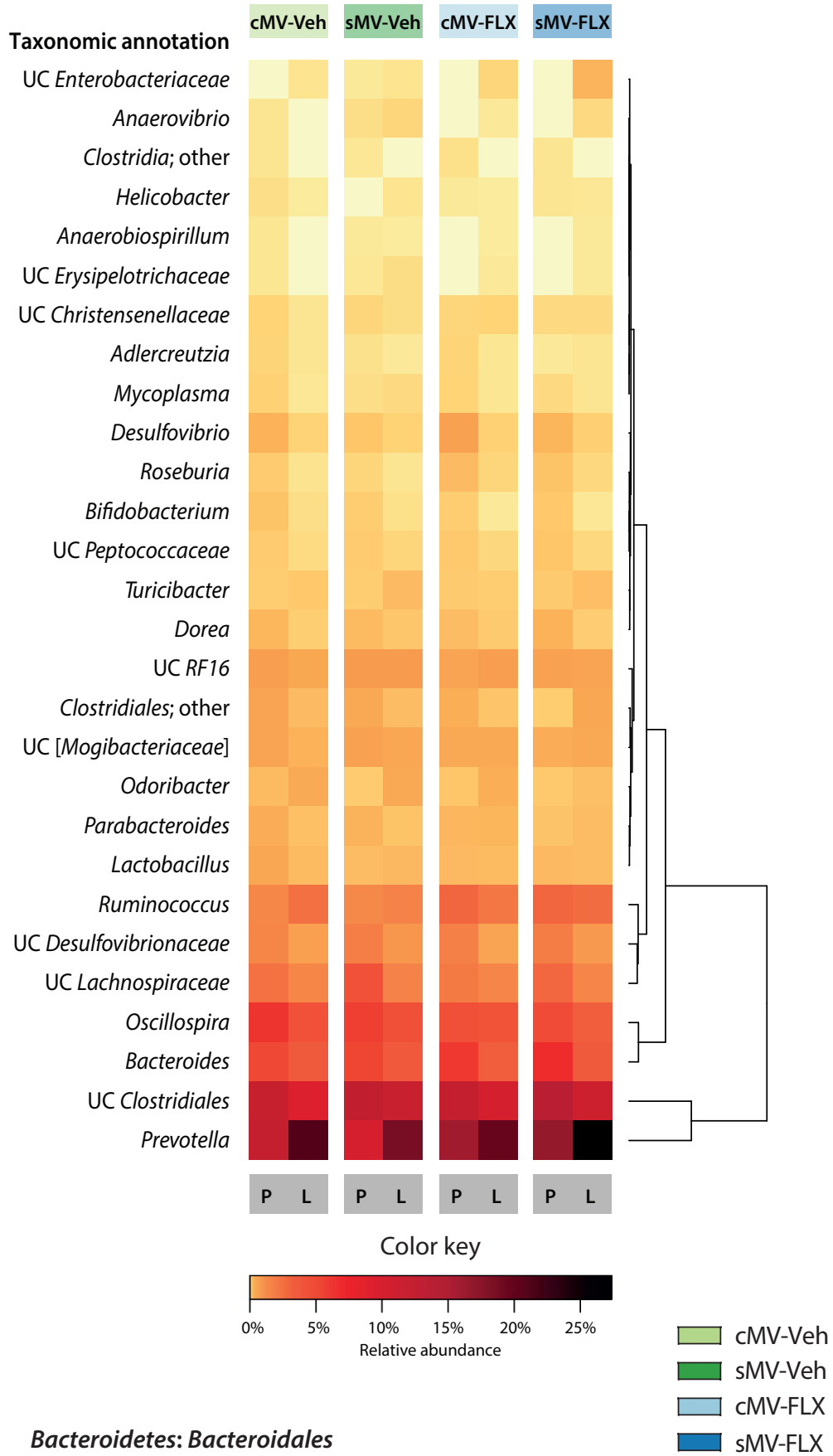

Ruminococcus

Bacteroides
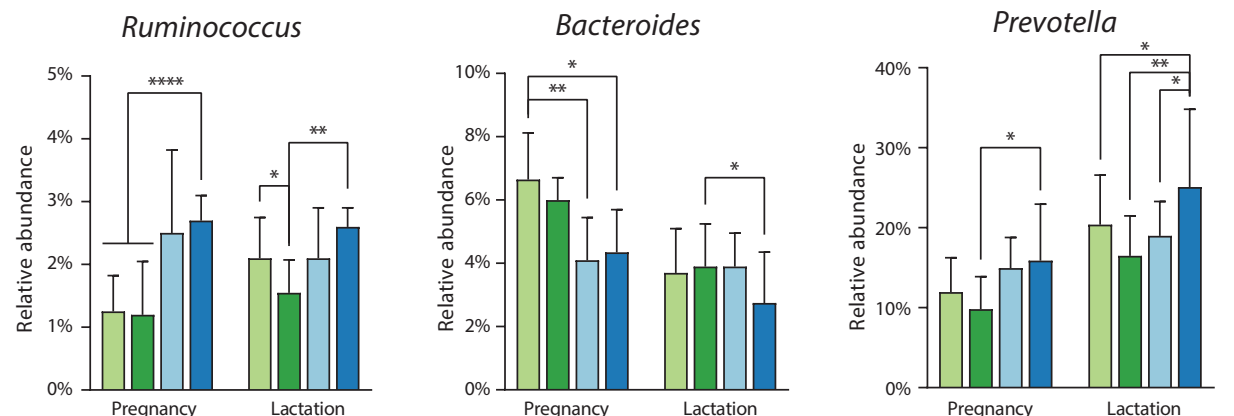

Parabacteroides

Odoribacter
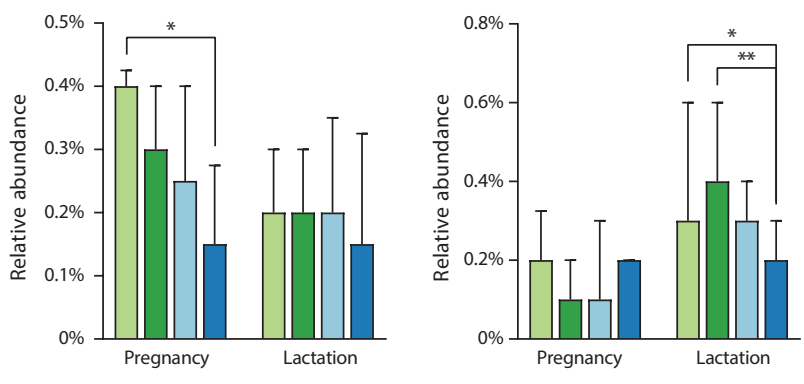
C Metabolites cMV-Veh sMV-Veh cMV-FLX sMV-FLX

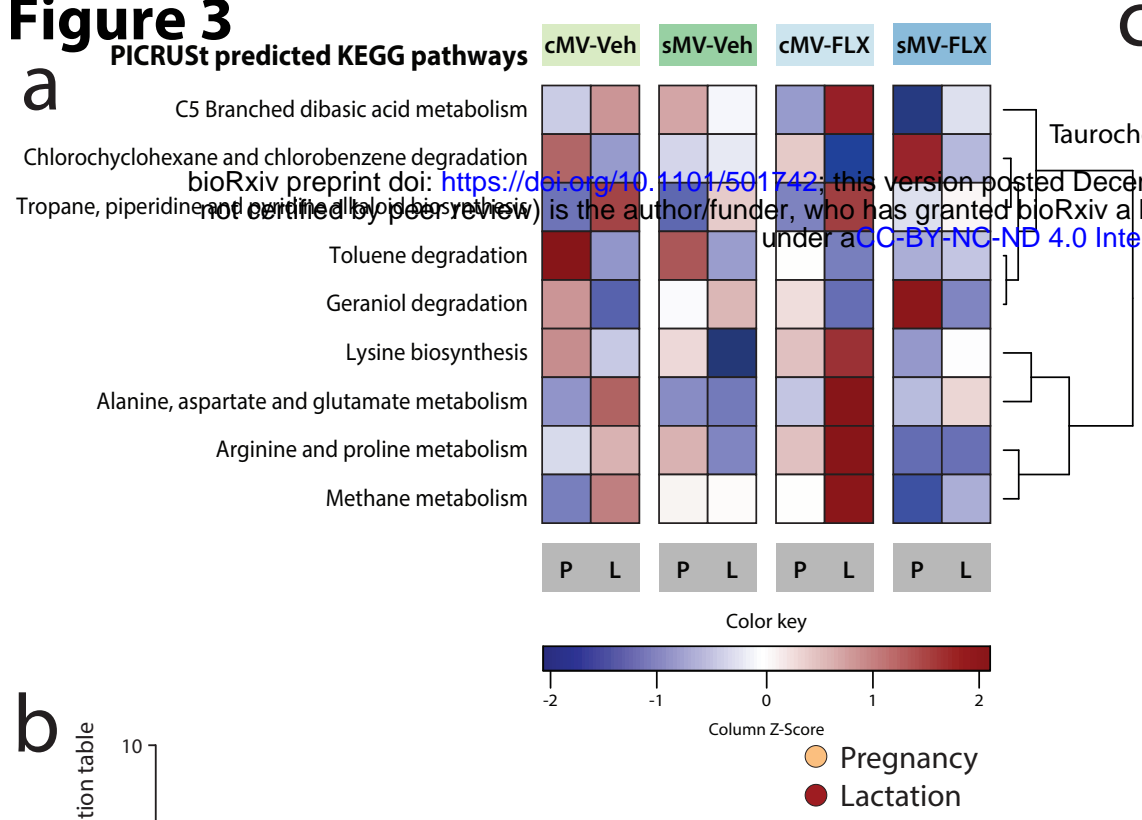

exane and chlorobenzene degradation

bioRxiv preprint doi: https://d

Toluene degradation

Geraniol degradation

Lysine biosynthesis

Arginine and proline metabolism

Methane metabolism

- Lactation

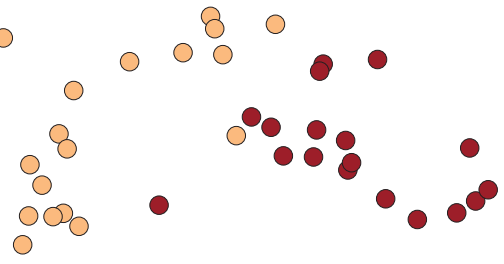

0

$-10$

PC1 (35.8\%) from metabolomics concentration table

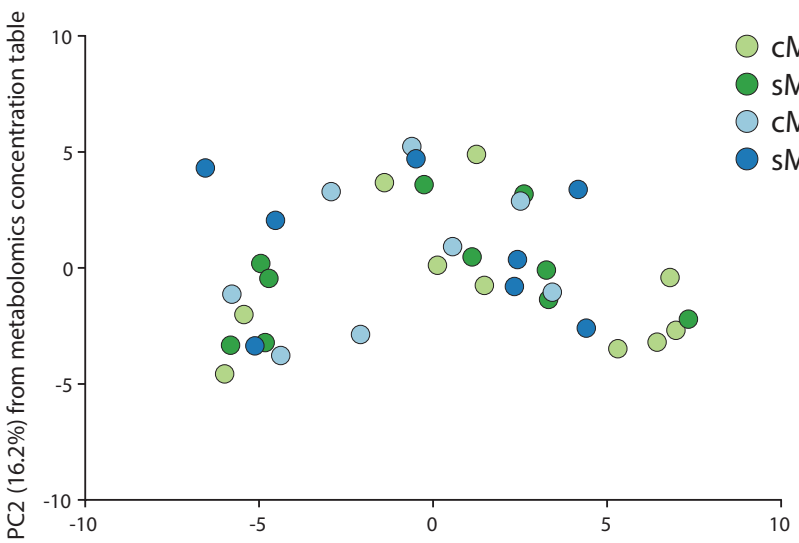

PC1 (35.8\%) from metabolomics concentration table

e

cMV-Veh pregnancy vs lactation

Homocysteine Degradation thanolamine Biosynthesis Sphingolipid Metabolism Selenoamino Acid Metabolism Methionine Metabolism Betaine Metabolism

Glycine and Serine Metabolism

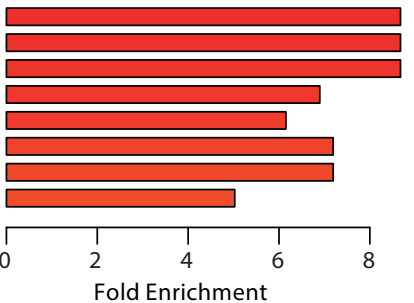

sMV-Veh pregnancy vs lactation
Ammonia Recycling Methionine Metabolism Homocysteine Degradation lethanolamine Biosynthesis Sphingolipid Metabolism Glycine and Serine Metabolism
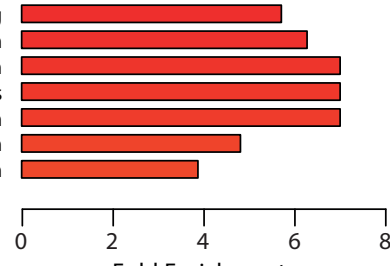

Fold Enrichment

CMV-FLX pregnancy vs lactation

No significantly altered metabolic pathways

sMV-FLX pregnancy vs lactation Glutamate Metabolism
cMV-Veh

sMV-Veh

cMV-FLX

d
Valine

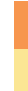

\section{nodeoxycholic acid}

Serine

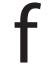

$f$

Acetic acid Isobutyric acid Glutamic acid

Alanine

Isoleucine

Valine

Glycine

Lysine

Threonine

Methionine

Serine

Aspartic acid

Isocaproic acid

Taurochenodeoxycholic acid

Valeric acid

Deoxycholic acid

Proline

Tyrosine

Phenylalanine

Butyric acid

Isovaleric acid

Betamuricholic acid

Omegamuricholic acid

Alphamuricholic acid

Glycocholic acid
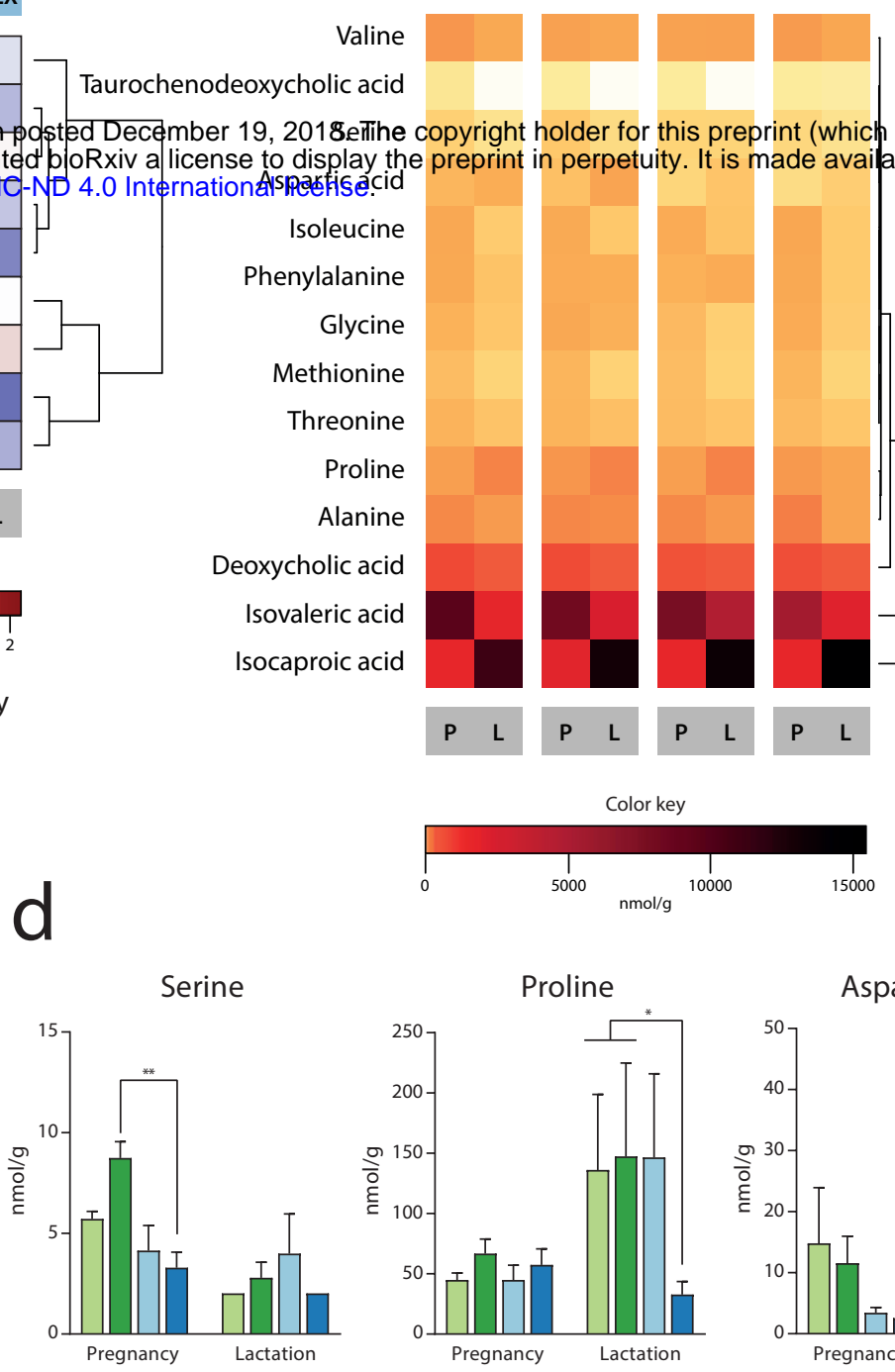

Aspartic acid
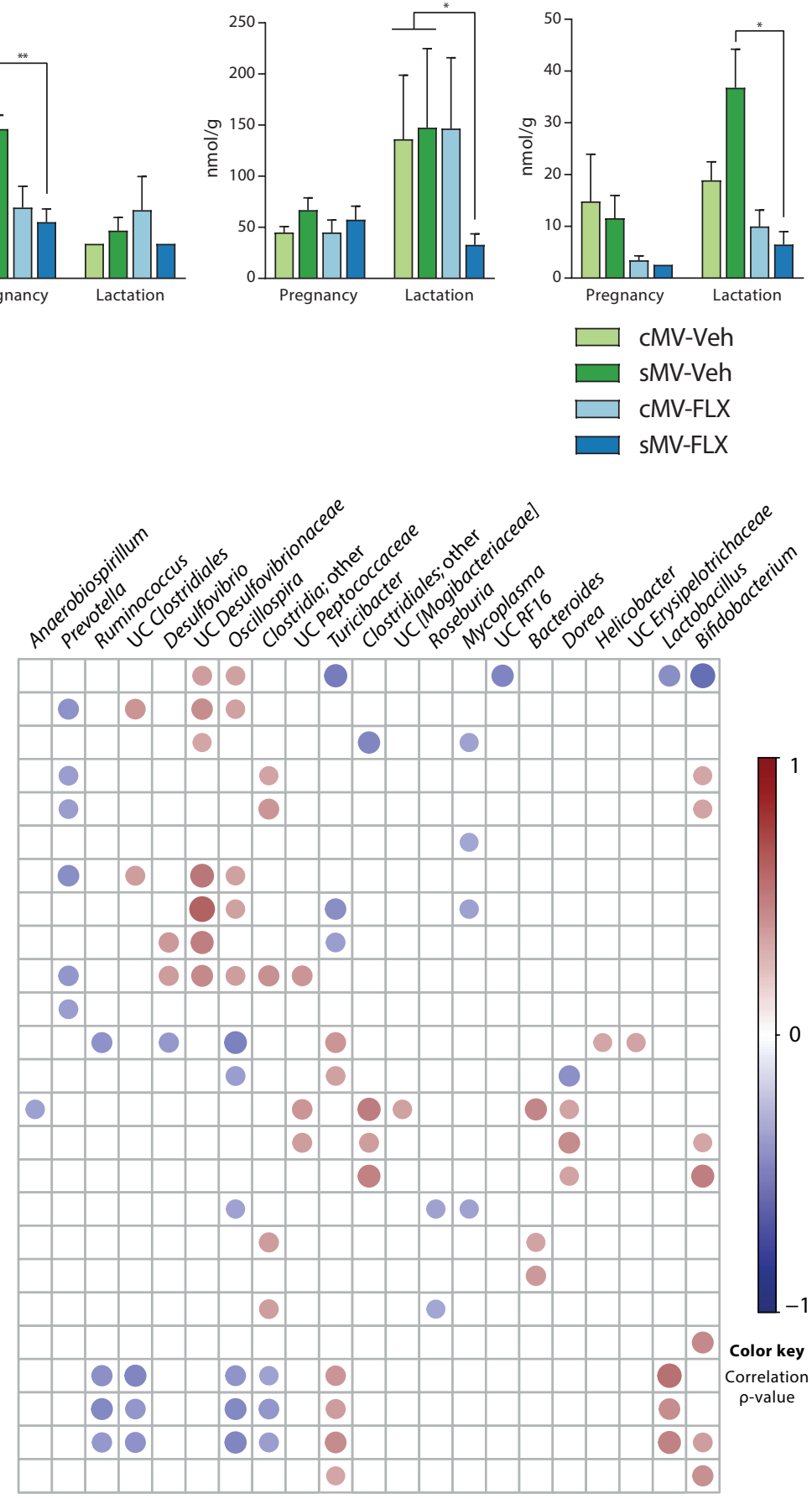
Supplemental Figure 1

a

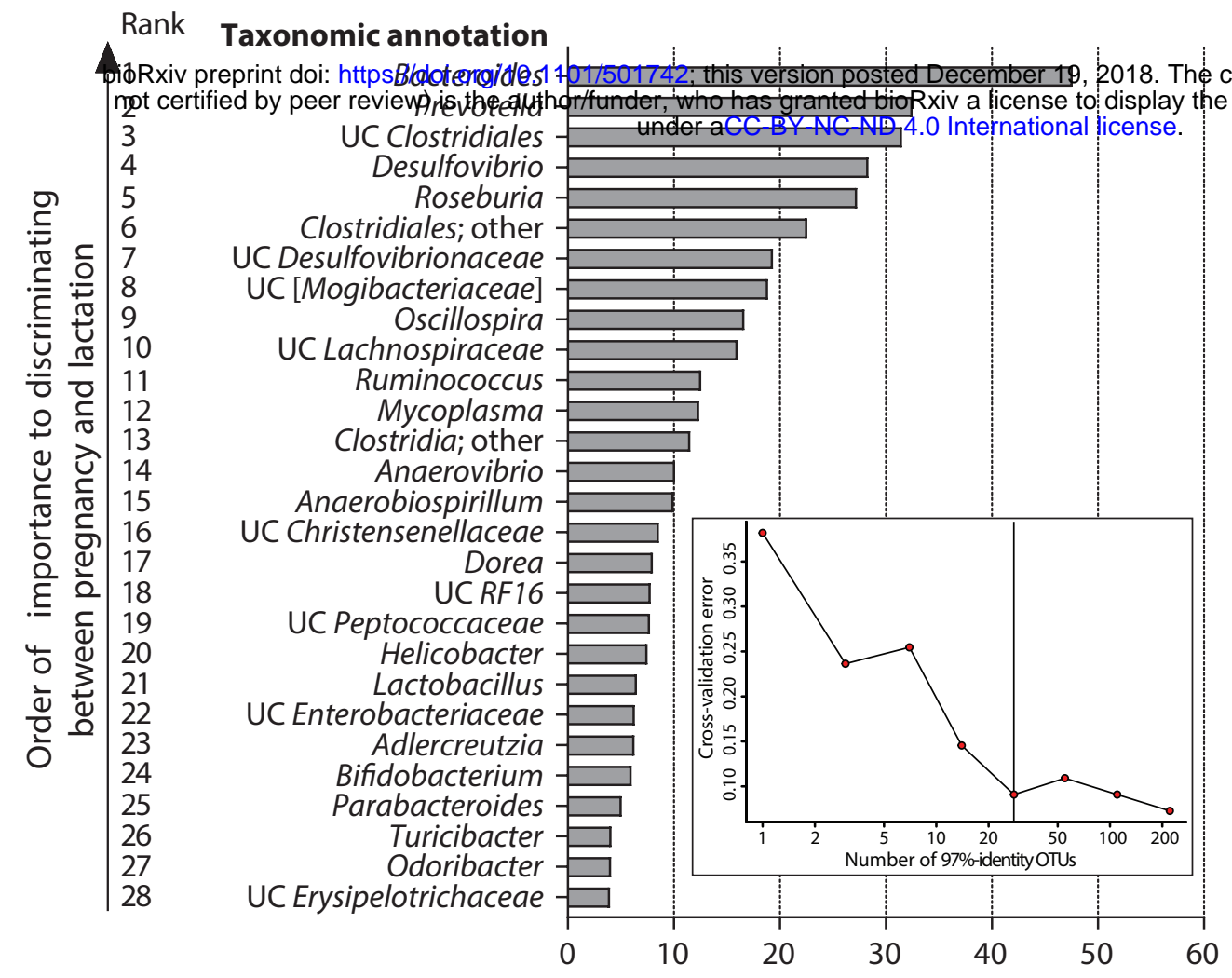

Mean decrease in accuracy if removed from model (\%) b

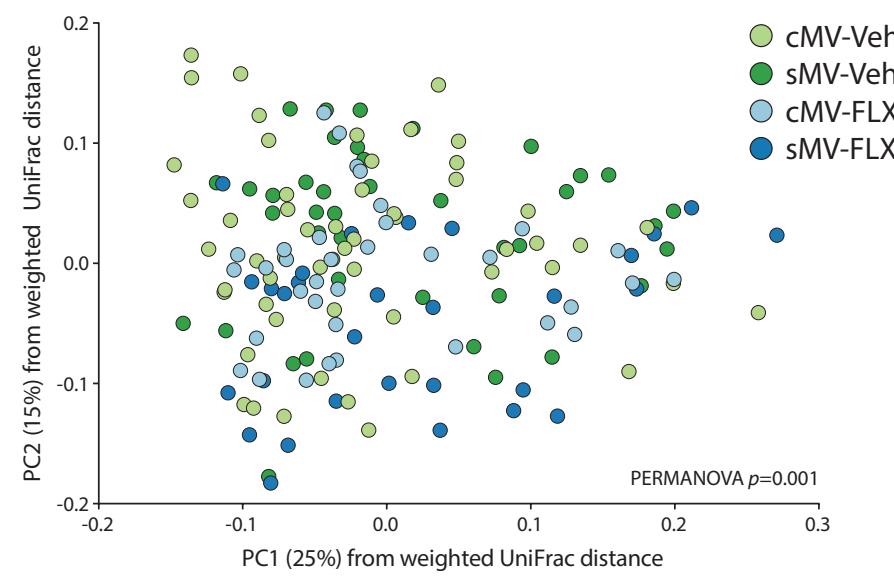

\section{C}

\section{Actinobacteria: Bifidobacteriales}

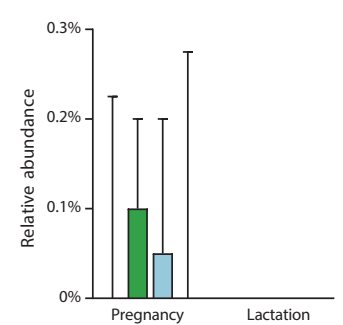
Bifidobacterium
Actinobacteria: Coriobacteriales

Adlercreutzia

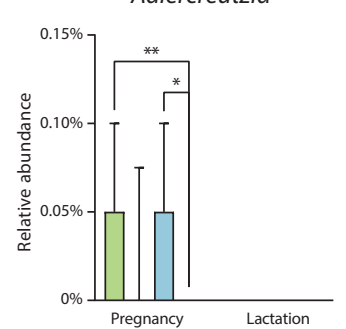

UC Lachnospiraceae

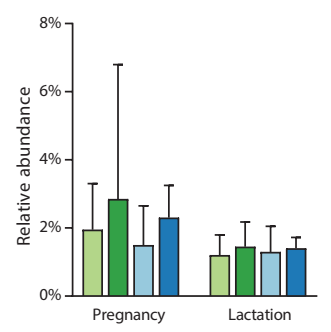

Bacteroidetes: Bacteroidales

UC RF16

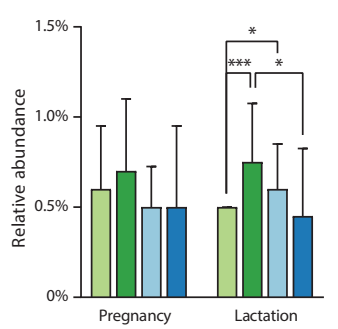

UC Christensenellaceae

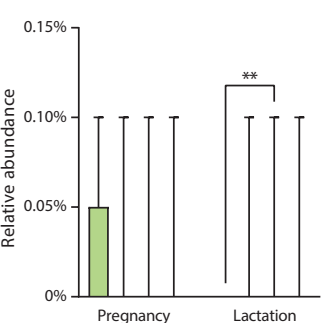

Firmicutes: Clostridiales

UC Clostridiales

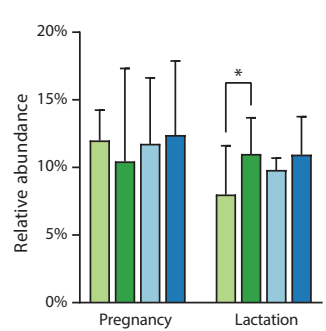

Clostridiales; other

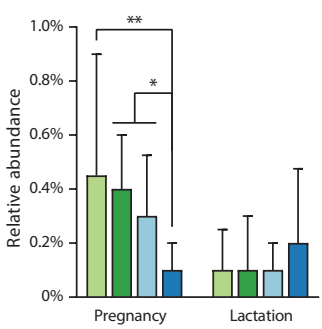

Firmicutes: Clostridiales
UC [Mogibacteriaceae]

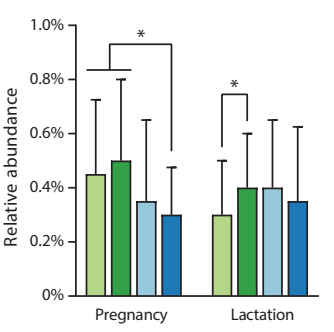

Oscillospira

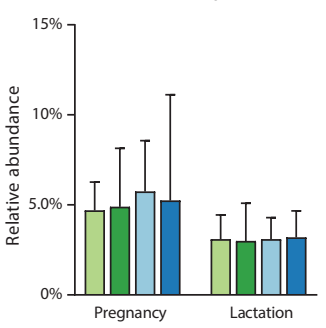

UC Peptococcaceae

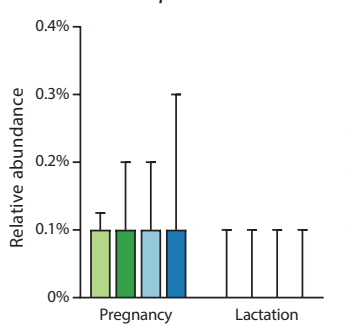

Roseburia

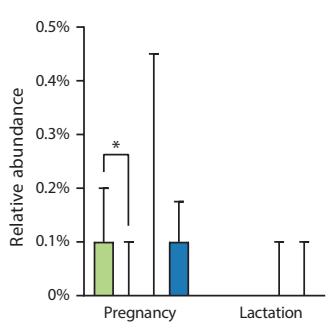


Firmicutes:

Lactobacillales

Firmicutes:

Turicibacterales

Proteobacteria: Desulfovibrionales

Tenericutes: Mycoplasmatales

bioRxiy preprint doi: https://doi.org/10.1101/501742; this version posted December 19.2018. The copyright holder for this preprint (which was
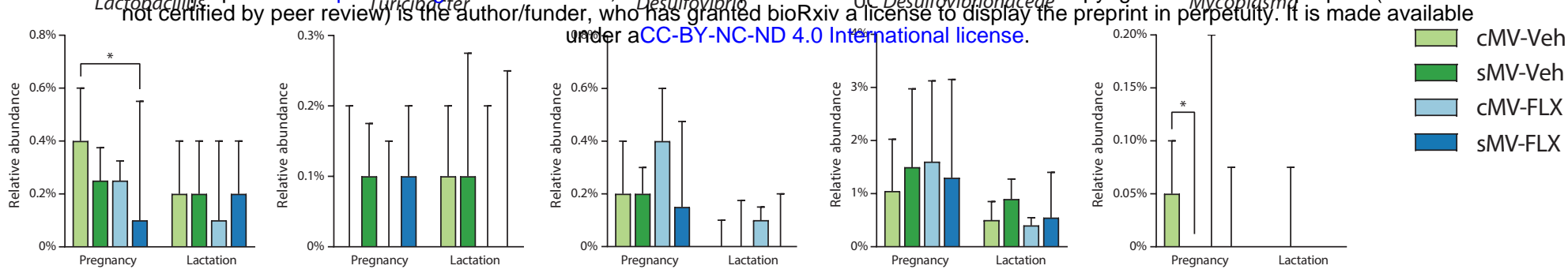

d

Desulfovibrio
UC Desulfovibrionaceae
UC Clostridiales
Oscillospira
Roseburia
UC Lachnospiraceae
Clostridia; other
UC Christensenellaceae
Dorea
Parabacteroides
Clostridiales; other
UC [Mogibacteriaceae $]$
UC RF16
UC Erysipelotrichaceae
Bacteroides
UC Peptococcaceae
Anaerobiospirillum
Adlercreutzia
Mycoplasma
Helicobacter
UC Enterobacteriaceae
Lactobacillus
Bifidobacterium
Turicibacter
Prevotella
Ruminococcus
Anaerovibrio
Odoribacter

Desulfovibrio

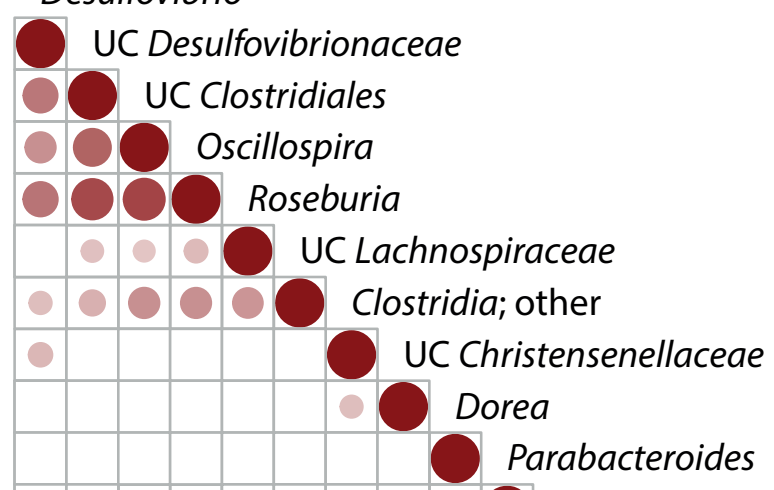


a bioRxiv preprint doi: https://doi.org/10.1101/501742; this Dsion posted December 19, 2018. The copyright holder for this Bacteroidales

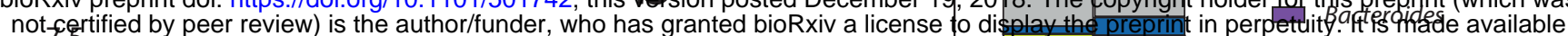

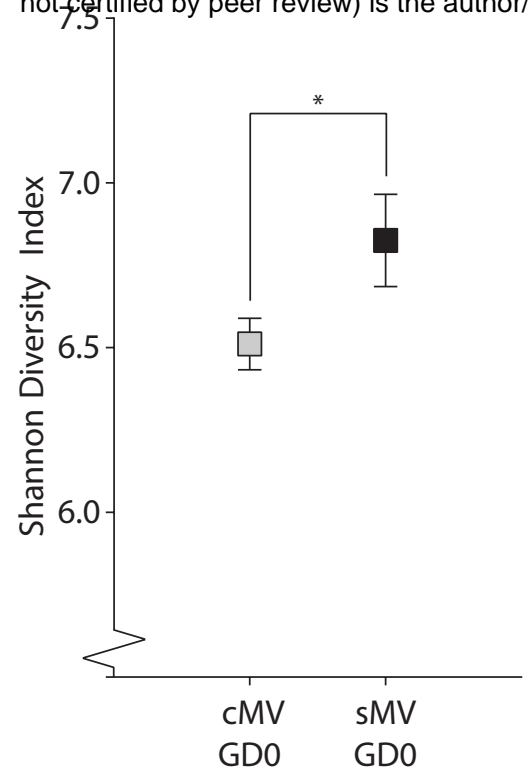
under aCC-BY-NC-ND 4.0 alntich

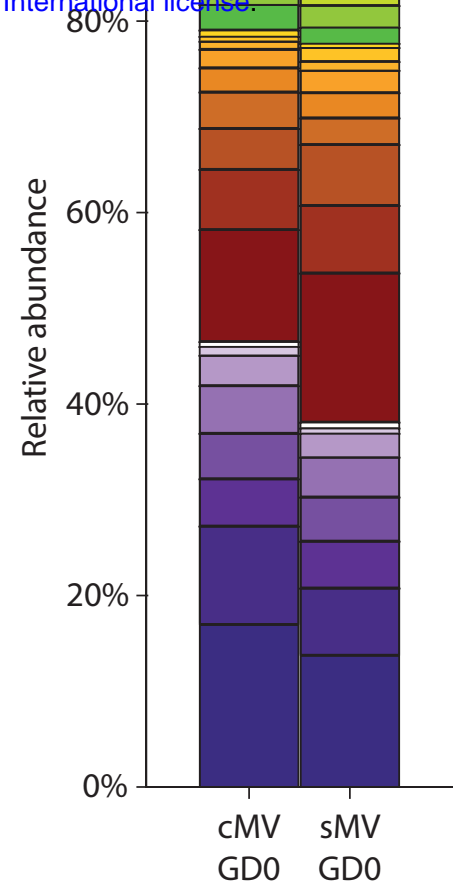

$\square$ UC S24-7

$\square$ UCRF16

$\square$ UC Rikenellaceae

Firmicutes

$\square$ UC Clostridiales

UC Ruminococcaceae

$\square$ Oscillospira

$\square$ Phascolarctobacterium

$\square$ Ruminococcus

$\square$ UC Lachnospiraceae

$\square$ Coprococcus

$\square$ UC Veillonellaceae

$\square$ UC Clostridiaceae

Proteobacteria

$\square$ Sutterella

$\square$ UC Desulfovibrionaceae

$\square$ UC Helicobacteraceae

Spirochaetes

$\square$ Treponema

Other

$\square$ Other

GDO GDO 

bjoRxiv preprint doi: https://doi.org/10.1101/501742; this version posted December 19, 2018. The copyright holder for this preprint (which was
Amino aciфist certified by peer review) is the author/funder, who has granted bioRxiv a license to display the preprint in perpetuity. It is made available

Glutamic acid

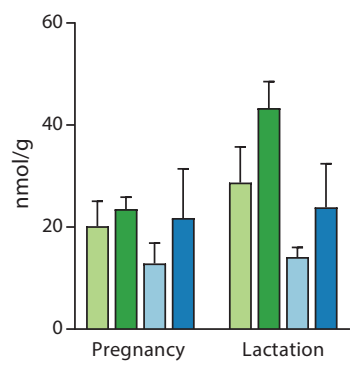

Bile acids

Alphamuricholic Acid

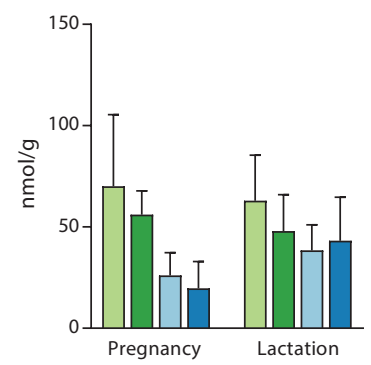

\section{Short-chain fatty acids}

Acetic Acid

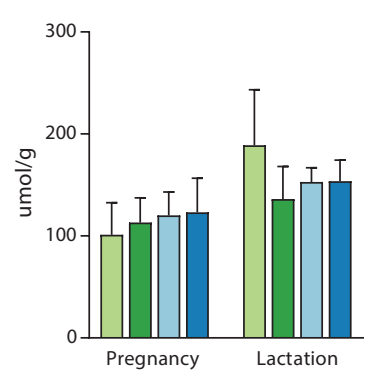

Lysine

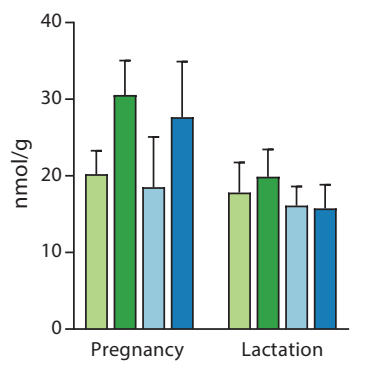

Betamuricholic Acid

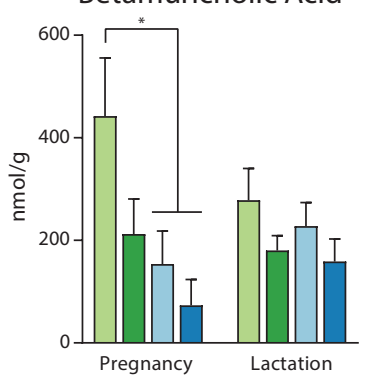

Isobutyric Acid

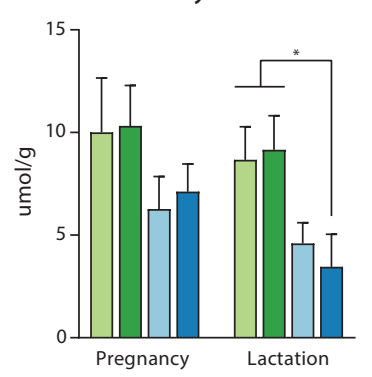

Tyrosine
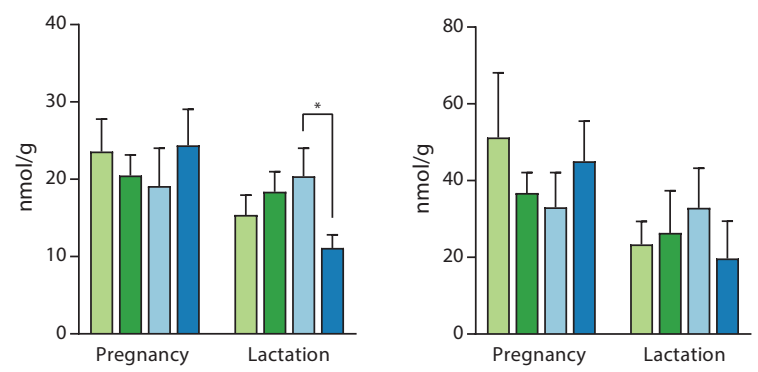

Lithocholic Acid
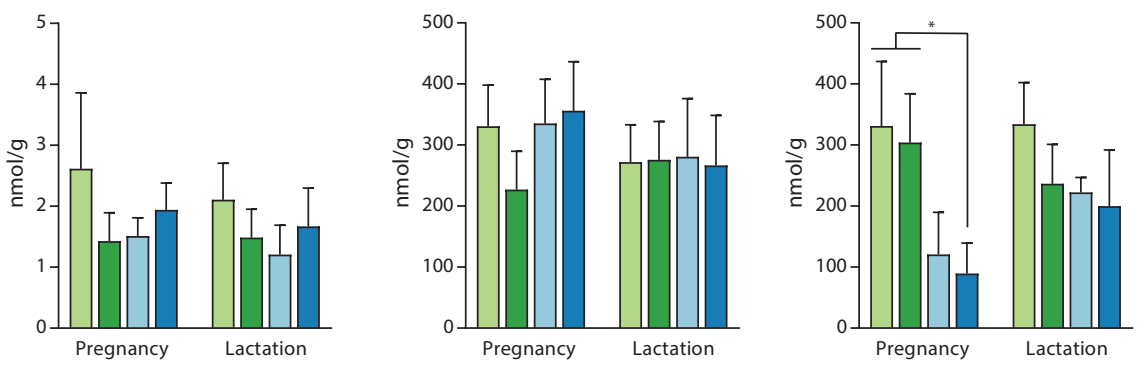

Butyric Acid

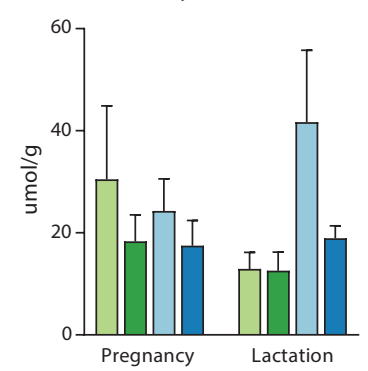

Valeric Acid

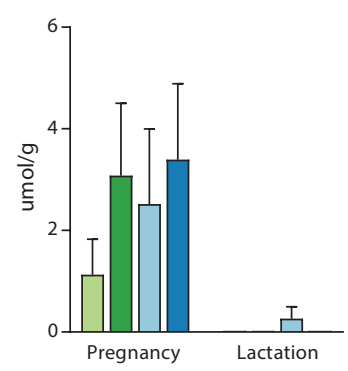

cMV-Veh

sMV-Veh

sMV-FLX
CMV-FLX 
Homocysteine Degradation

Phosphatidylethanolamine Biosynthesis

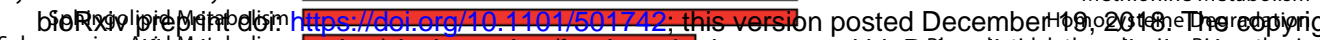

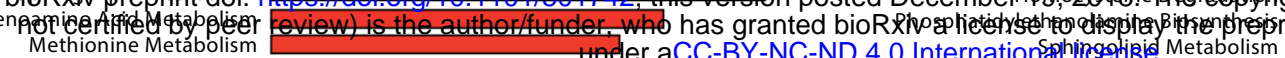
Betaine Metabolism

permidine and Spermine Biosynthesis

Glycine and Serine Metabolism Ammonia Recycling Threonine and 2-Oxobutanoate Degradation Valine, Leucine and Isoleucine Degradation Bile Acid Biosynthesis

lutathione Metabolism

Glucose-Alanine Cycle

Tryptophan Metabolism

Glutamate Metabolism

Phenylalanine and Tyrosine Metabolism

Porphyrin Metabolism

Catecholamine Biosynthesis

Thyroid hormone synthesis Urea Cycle

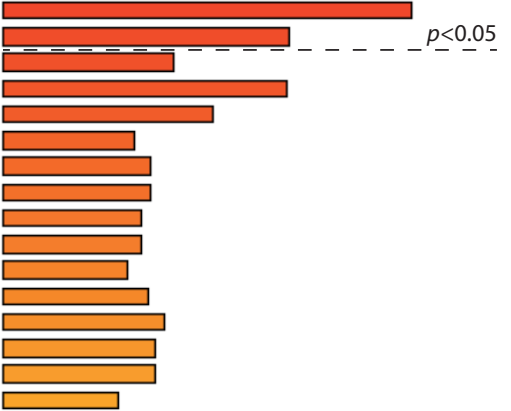

Glycine and Serine Metabolism Glutamate Metabolism Urea Cycle Betaine Metabolism Spermidine and Spermine Biosynthesis Purine Metabolism Selenoamino Acid Metabolism Lysine Degradation

Beta-Alanine Metabolism Malate-Aspartate Shuttle Tyrosine Metabolism Arachidonic Acid Metabolism Cysteine Metabolism Folate Metabolism Histidine Metabolism Nicotinate and Nicotinamide Metabolism Warburg Effect Aspartate Metabolism

Propanoate Metabolism Arginine and Proline Metabolism Purine Metabolism Carnitine Synthesis Tyrosine Metabolism Aspartate Metabolism Ethanol Degradation Pyruvate Metabolism Beta-Alanine Metabolism Malate-Aspartate Shuttle Amino Sugar Metabolism Fatty Acid Biosynthesis Butyrate Metabolism Arachidonic Acid Metabolism

Cysteine Metabolism Folate Metabolism Histidine Metabolism amide Metabolism Warburg Effect Lysine Degradatio Biotin Metabolism

$p$ value

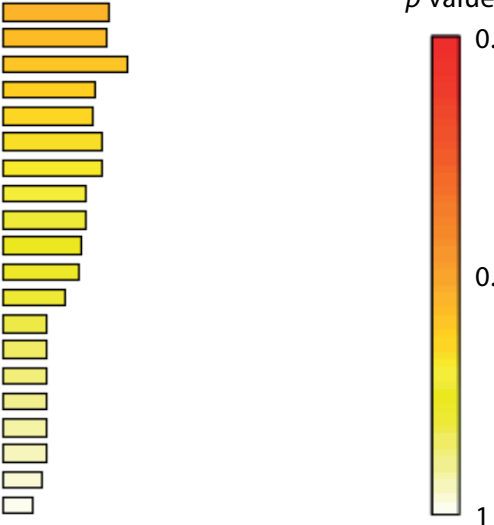

\begin{tabular}{|c|c|c|c|}
\hline Г & $T$ & T & $\mathrm{T}$ \\
\hline 0 & 2 & 4 & 6 \\
\hline
\end{tabular}

Fold Enrichment

\section{cMV-FLX group}

$p<0.05$

Betaine Metabolism ermine Biosynthesis Purine Metabolism Bile Acid Biosynthesis Glutamate Metabolism Aspartate Metabolism Arginine and Proline Metabolism

Methionine Metabolism Beta-Alanine Metabolism Malate-Aspartate Shuttle Porphyrin Metabolism Glycine and Serine Metabolism

Tyrosine Metabolism Urea Cycle

Ammonia recycling

Ethanol Degradation

Pyruvate Metabolism

Alanine Metabolism

Glutathione Metabolism Carnitine Synthesis

Cacose-Alanine Cycle

ryptophan Tryptophan Metabolism Amino Sugar Metabolism ne and Isoleucine Degradation Arachidonic Acid Metabolism

Cysteine Metabolism

Folate Metabolism Histidine Metabolism mide Metabolism Nicotinate and Nicotinamide Metabolism
Warburg Effect
Selenoamino Acid Metabolism Selenoamino Acid Metabolism
Fatty Acid Biosynthesis

Butyrate Metabolism

Catecholamine Biosynthesis

Thyroid hormone synthesis

Homocysteine Degradation Phosphatidylethanolamine Biosynthesis Sphingolipid Metabolism Biotin Metabolism Lysine Degradation

Propanoate Metabolism Phenylalanine and Tyrosine Metabolism

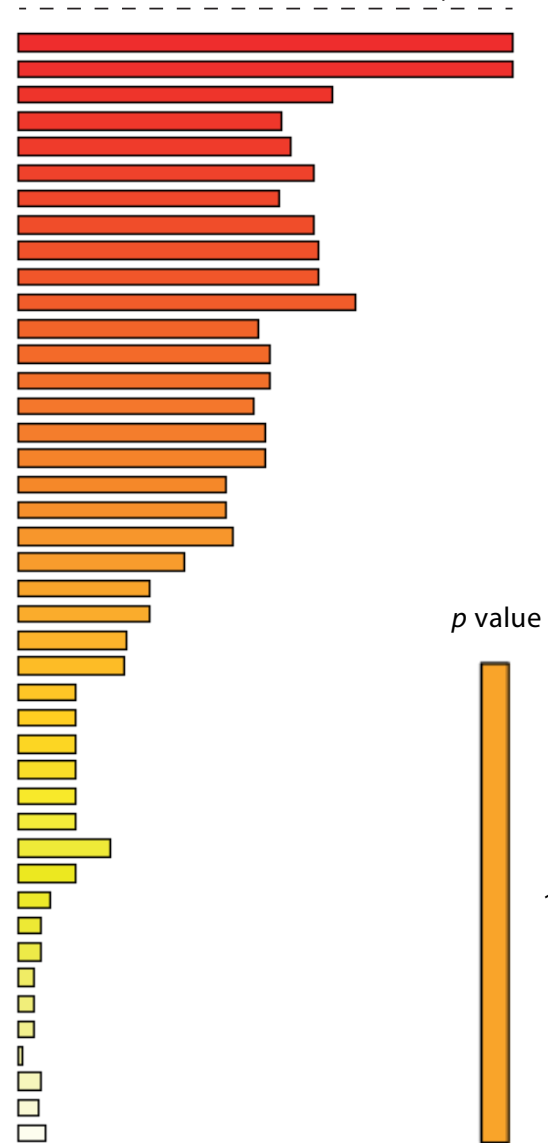

sMV-FLX group

Spermidine and Spermine Biosynthesis Selenoamino Acid Metabolism Catecholamine Biosynthesis Thyroid hormone synthesis

Methionine Metabolism Glycine and Serine Metabolism Urea Cycle

Phenylalanine and Tyrosine Metabolism Glutamate Metabolism Valine, Leucine and Isoleucine Degradation Glucose-Alanine Cycle Tryptophan Metabolism Alanine Metabolism Glutathione Metabolism Porphyrin Metabolism Homocysteine Degradation Phosphatidylethanolamine Biosynthesis Sphingolipid Metabolism Carnitine Synthesis Biotin Metabolism

Arginine and Proline Metabolism Threonine and 2-Oxobutanoate Degradation Bile Acid Biosynthesis Ammonia Recycling Tyrosine Metabolism Purine Metabolism Ethanol Degradation Pyruvate Metabolism Propanoate Metabolism Beta-Alanine Metabolism Malate-Aspartate Shuttle Fatty Acid Biosynthesis Aspartate Metabolism

Lysine Degradation

Butyrate Metabolism Arachidonic Acid Metabolism

Cysteine Metabolism

Folate Metabolism

Histidine Metabolism Nicotinate and Nicotinamide Metabolism Warburg Effect $\square$

Amino Sugar Metabolism $\square$

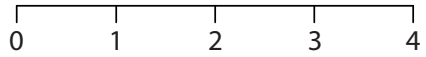

Fold Enrichment 